\title{
Taurine reprograms mammary-gland metabolism and alleviates inflammation induced by Streptococcus uberis
}

\section{Riguo Lan}

Nanjing Agricultural University

\section{Zhixin Wan}

Nanjing Agricultural University

Yuanyuan Xu

Nanjing Agricultural University

Zhenglei Wang

Nanjing Agricultural University

Shaodong Fu

Nanjing Agricultural University

Yuanyuan Zhou

Nanjing Agricultural University

Xinguang Lin

Nanjing Agricultural University

Xiangan Han

Chinese Academy of Agricultural Sciences

Zhenhua Luo

Cranfield University https://orcid.org/0000-0003-0766-6174

Jinfeng Miao ( $\triangle$ miaojinfeng@njau.edu.cn )

Nanjing Agricultural University

\section{Yulong Yin}

Chinese Academy of Sciences

\section{Article}

Keywords: mastitis, Streptococcus uberis, taurine, metabolic regulation, AMPK, mTOR

Posted Date: March 16th, 2021

DOI: https://doi.org/10.21203/rs.3.rs-289639/v1 
License: (c) (i) This work is licensed under a Creative Commons Attribution 4.0 International License. Read Full License 


\section{Abstract}

Streptococcus uberis (S. uberis) is an important pathogen causing mastitis,which causes continuous inflammation and dysfunction of mammary glands and leads to enormous economic losses. Most research on infection continues to be microbial metabolism-centric, and many overlook the fact that pathogens require energy from host. In this perspective, we uncover metabolic reprogramming during host immune responses is associated with infection-driven inflammation, particularly when caused by intracellular bacteria. Taurine, a metabolic regulator, has been shown to effectively ameliorate metabolic diseases. We evaluated the role of taurine in the metabolic regulation of $S$. uberis-induced mastitis. Metabolic profiling indicates that $S$. uberis exposure triggers inflammation and metabolic dysfunction of msmmary glands and mammary epithelial cells (MECs, the main functional cells in mammary glands). Challenge with $S$. uberis upregulates glycolysis and oxidative phosphorylation in MECs. Pretreatment with taurine restores metabolic homeostasis, reverses metabolic dysfunction by decrease of lipid, amino acid and especially energy disturbance in the infectious context, and alleviates excessive inflammatory responses. These outcomes depend on taurine-mediated activation of the AMPK-mTOR pathway, which inhibits the over activation of inflammatory responses and alleviates cellular damage. Thus, metabolic homeostasis is essential for reducing inflammation. Metabolic modulation can be used as a prophylactic strategy against mastitis.

IMPORTANCE: Mastitis, especially caused by Streptococcus uberis, induces continuous inflammation and dysfunction of mammary glands, leading to enormous economic losses in dairy industry. Mammary epithelial cells (MECs) are mammary gland-resident major functional cells which occupy a significant role in maintaining homeostasis in mammary glands. MECs can adopt an inflammatory trending in response to $S$. uberis infection and try to clear these intracellular bacteria. Researchers at Nanjing Agricultural University in China found that $S$. uberis induces MECs acute inflammation caused by metabolic dysregulation, that is, increased oxidative phosphorylation (OXPHOS) and glycolysis. However, taurine pretreatment can enhance metabolic fitness and anti-inflammatory activity. As a potential therapy, metabolic regulation with taurine restored MECs homeostasis and reduced excessive inflammation. The findings suggest that modulating MECS metabolism might be a new therapeutic strategy for mastitis as well as other bacterial infections.

\section{Introduction}

Mastitis is a highly prevalent and important infectious disease worldwide. More than 150 different pathogens can cause mammary gland infection and no specific vaccines are available. Antibiotics combined with anti-inflammatory drug therapy is the usual method to control mastitis (1). These therapies are often ineffective for the diversity of pathogens and the complexity of breast tissue $(2,3)$. Milk production of therapeutically treated cows will often decline significantly during subsequent lactations necessitating culling of treated animals. Moreover, the extensive use of antibiotics in the treatment and control of mastitis has implications for human health via residual drugs and increased emergence of antibiotics-resistant bacteria strains then enter the food chain (4). Mastitis leads to great 
economic losses to the dairy industry and poses serious potential threats to public health. Clearly, new and innovative approaches for mastitis control are needed.

S. uberis accounts for approximately 33 per cent of the isolates obtained from clinical cases of bovine mastitis (5). Growing evidence suggests that it can also infect humans thus directly threatening human health (6). Mastitis induced by $S$. uberis is difficult to control because $S$. uberis may internalize and persist in mammary cells to develop an intracellular infection, thereby escaping detection and subsequent elimination by the host immune system and/or antibiotics (7). In the past decade, our lab engaged in elucidation of pathogen infection and host defense mechanisms of mastitis (8-10). We found that immunophysiological regulators (amino acids, polysaccharide, vitamins, and antioxidants, etc.) can reduce inflammatory damage of mammary tissues by improving the disease resistance of the host animal (8-12). Physiological regulation of the immune system has potential as a putative therapeutic and prophylactic strategy for mastitis control with promising clinical applications.

Taurine is one of the most abundant free amino acids in most animal tissues and plays an important role in several essential biological processes. Our previous studies have established that taurine administration can modulate many mammary infections including those due to $S$. uberis $(11-13)$. We wonder how cells sense nutrient signaling from taurine and change it to an anti-infection effect in vitro and in vivo. Taurine downregulates $S$. uberis induced inflammation and decreases intracellular bacteria through the PI3K/Akt/mTOR, PIs/PLCY/DG/PKCa, and IP3/Ca ${ }^{2+} / \mathrm{CaM} / \mathrm{CaN}$ pathways by activating tolllike receptors (TLRs) $(13,14)$. Taurine also upregulates autophagy of mammary epithelial cells (MECs) during $S$. uberis infection (15). We postulate that taurine attenuates $S$. uberis-induced inflammation through its regulatory effect on metabolism.

Host metabolic homeostasis is critical to disease progression during pathogen infection, as intracellular bacteria often reprogram host metabolism to divert nutrients for their own growth. Although pathogenic microorganisms also change their metabolic pathways to facilitate invasion, the metabolic disorders of host are often closely related to diseases. For example, Mycobacterium tuberculosis increases glucose uptake and decreases oxidative phosphorylation (OXPHOS) to redirect glycolytic intermediates toward lipid synthesis, which facilitates bacterial growth within macrophages $(16,17)$. Metabolic intervention can be used to combat pathogenic infections. Wang et al. show that limiting glucose utilization significantly improves tolerance to cerebral malaria (18). In vivo, taurine participates in the synthesis of fatty acids and steroids (19). It can be converted into taurocholic acid and participate in bile formation thus promoting the emulsification and absorption of lipids (19). It can produce L-alanine and L-glutamate via transaminase. Taurine may therefore influence $S$. uberis infection via regulation of cell metabolism.

Metabolic homeostasis is primarily regulated by the precise coordination of glycolysis, the TCA cycle, the pentose phosphate pathway, fatty acid oxidation, fatty acid synthesis, and amino acid metabolism. Recently, it had been discovered that various immune cells undergo distinct metabolic changes in the face of pathogenic infections. These mechanisms require an increased energy expenditure and the consumption of intermediate metabolites (20-22). M1 macrophages, T helper 1 (TH1) and TH17 cells, 
upregulate glycolysis which helps define their inflammatory phenotype (23). M2 macrophages as well as quiescent and regulatory $T$ cells, may be characterized as an anti-inflammatory phenotype depending on OXPHOS and fatty acid oxidation for tissue repair and anti-inflammatory cytokine production (24). Mammary epithelial cells (MECs) are responsible for milk synthesis. It has been established that they are involved in innate immune and anti-infection responses $(25,26)$. The metabolic changes and underlying mechanisms of MECs as a result of bacterial infection remain unclear.

AMP-dependent protein kinase (AMPK) is an "energy receptor" that regulates energy metabolism in eukaryotic cells (27). mTOR is a "hub" that regulates metabolism and is targeted by AMPK activation (28). These molecules communicate in the metabolism of substances and energy. Whether taurine regulates the metabolism of the host through them and affects the redistribution of cellular material and energy in the mammary glands, thereby regulating the infection of $S$. uberis, is of interest in our laboratory. Herein, we provide insight into the metabolic changes of mammary glands/cells in $S$. uberis infection and the regulatory mechanism(s) of taurine in this bioprocess. Our results contribute toward developing novel prophylactic strategies for mastitis and possibly other intracellular infections.

\section{Results}

\section{Taurine reprograms $S$. uberisinduced metabolic changes in mammary glands}

To determine whether taurine regulates metabolism in $S$. uberis induced mastitis, we infected the teats of taurine-pretreated mice with $S$. uberis for $24 \mathrm{~h}$ and then collected mouse mammary glands for metabolomic analysis. As shown in Fig. S1, intragastric administration of taurine alone could partly change mammary gland metabolism in mice. The metabolites increased by taurine administration focused on amino acids (such as proline, isoleucine, tyrosine, L-cysteine, L-allothreonine), nucleic acids (xanthine, uridine monophosphate), and taurine-associated metabolite sulfuric acid. These metabolites mainly concentrated on aminoacyl-tRNA biosynthesis, sulfur metabolism, purine metabolism, valine, leucine and isoleucine biosynthesis, and cysteine and methionine metabolism (Fig. S1). Further, taurine administration significantly changed the metabolites in S. uberis-infected mammary glands (Fig. S2). S. uberis-infected mammary glands exhibited 52 different metabolites, including 7 upregulated carbohydrate-related metabolites (galactose, mannose, galactinol, N-acetyl-D-galactosamine, 6phosphogluconate, glucoheptonate, ribose, and 5'-methylthioadenosine) and 7 amino acids or aminoacid derivatives (valine, proline, serine, leucine, L-allothreonine, creatine, and 3, 5-dihydroxyphenylglycine). 1 TCA-cycle intermediate (fumarate), 3 amino-acid metabolites (cysteine, ornithine and ascorbate) and 3 fatty acid-metabolism precursors (glycerate, 2-monpalmitin and palmitic acid) are down-regulated (Fig. 1A). These metabolites were primarily involved in the pentose phosphate pathway; pantothenate and CoA biosynthesis; arginine and proline metabolism; glycine, serine, and threonine metabolism; valine, leucine, and isoleucine biosynthesis; glutathione metabolism and aminoacyl-tRNA biosynthesis (Fig. 1B). Taurine pretreatment decreased glycerol, stearic acid, capric acid and 2-hydroxybutanoate levels, elevated several lipid metabolites (palmitic acid, lignoceric acid, and $\beta$-glycerophosphoric acid) (Fig. 1C), and influenced unsaturated fatty-acid and fatty-acid biosynthesis, fatty-acid elongation, and purine and 
pyrimidine metabolism (Fig. 1D). Integrated metabolite-map and pathway analysis showed lower metabolite levels of the TCA cycle (fumarate) and glutathione metabolism (cysteine, ornithine and ascorbate), along with higher metabolite levels in the pentose phosphate pathway and higher amino acid levels following $S$. uberis infection. These data denoted consumption of more energy and production of anti-infectious metabolites (such as glutathione, an antioxidant) in mammary tissue to resist $S$. uberis challenge (Fig. 1E). Taurine pretreatment mainly reduced fatty acid metabolism (2-hydroxybutanoate), promoted unsaturated fatty acid and fatty acid biosynthesis, and nucleic acid metabolism (Fig. 1E). To confirm whether these metabolic changes were reflected at a transcriptional level, we measured the expression of key genes involved in glycolysis (HK, PFK1 and GAPDH), TCA cycle (PDH, SDH), fatty acid metabolism (PPAR-y, LIPA) and pentose phosphate pathway (G6PDH). In line with our findings, expression of these genes in mammary glands was elevated by $S$. uberis challenge. Taurine dropped off most of the gene expression in S. uberis infection but increased gene expression of G6PDH (Fig. 1F). These data suggest that taurine reprograms metabolism in $S$. uberis-infected mammary glands.

\section{Taurine attenuates metabolic disturbances in S. uberis challenged MECs}

MECs are the main functional cells in mammary glands and play important roles in the mammary gland defense system. Pretreatment with taurine alone changed taurine-associated metabolites in mouse mammary epithelial cell line (EpH4-Ev cells). $\beta$-alanine, a competitive inhibitor of taurine transporter (TauT), was decreased by taurine pretreatment. Differently, pretreatment with taurine increased intracellular taurine and taurine-associated metabolites (glutathione, sulfuric acid, etc.). These metabolites belonged to several taurine metabolism related pathways, including sulfur metabolism, taurine and hypotaurine metabolism, $\beta$-alanine metabolism, glutathione metabolism, and primary bile acid biosynthesis (Fig. S3).

To investigate whether taurine regulates MEC metabolism during S. uberis infection, EpH4-Ev cells were incubated with $S$. uberis for varying times and the intracellular metabolite profile was examined.

Metabolites in infected and control cells formed separate clusters on PCA and OPLA-DA plots (Fig. S4A-F) and had significant changes on volcano plots over time (Fig. S4G-H), especially at $3 \mathrm{~h}$ post-infection (Fig. S4I). Thus, challenge with $S$. uberis altered the cellular metabolic profile. Taurine pretreatment altered the metabolic profile of S. uberis-infected cells over these time points (Fig. S4J-R).

Metabolic changes induced by $S$. uberis infection at $1 \mathrm{~h}$ was slight and only several metabolites changed, but an increase of glucose-6-phosphate was present (Fig. S5A-E). In contrast, 2 or $3 \mathrm{~h}$ of infection with $S$. uberis upregulated 3 glycolysis-related metabolites (glucose, pyruvate and lactate), 4 lipid-related metabolites (octadecanol, 1-hexadecanol, 1-monopalmitin, and palmitic acid), 10 amino acids (alanine, valine, glycine, threonine, isoleucine, proline, serine, beta-alanine, taurine, and ornithine), and several other carbohydrate-related metabolites (fructose, tagatose, and inositol), while the levels of citrate and aketoglutarate were downregulated (Fig. 2A and Fig. S6A). The alterations at 2 or $3 \mathrm{~h}$ post-infection included the following metabolic pathways: aminoacyl-tRNA biosynthesis; TCA cycle; pyruvate metabolism; glycolysis or gluconeogenesis; valine; leucine, and isoleucine biosynthesis; pantothenate and 
CoA biosynthesis; glycine, serine, and threonine metabolism; arginine and proline metabolism; alanine, aspartate, and glutamate metabolism; and glutathione metabolism (Fig. 2B and Fig. S6B).

Pretreatment with taurine at $1 \mathrm{~h}$ post-infection exerted a slight change in amino acid metabolism (Fig. S5C-E). In S. uberis-infected cells ( 2 or $3 \mathrm{~h}$ ), taurine pretreatment decreased carbohydrate-related metabolite levels (glucose, pyruvate, lactate, citrate, a-ketoglutarate etc.) and amino acid levels (aspartate, alanine, isoleucine, $\beta$-alanine, valine, glutamate, proline), and attenuated their matched pathways (i.e., valine, leucine, and isoleucine biosynthesis; $\beta$-alanine, alanine, aspartate, and glutamate metabolism; and TCA cycle (Fig. 2C-E and Fig. S6C-E). These data suggest that taurine reprograms S. uberis-induced metabolic changes in MECs infected by $S$. uberis in a time dependent manner and the alterations are not totally matched with those in the mammary glands.

\section{MECs adopt distinct metabolism in response to various microbial stimuli compared to macrophages}

There are several cell types in mammary tissue including macrophages, polymorphonuclear neutrophilic leukocytes (PMN) and regulatory T cells (Treg). Metabolic reprogramming in most proinflammatory phenotype innate immune cells (i.e., dendritic cells, M1 macrophages, and NK cells) are characterized by elevated levels of TCA intermediates (succinate, citrate) and itaconate (An inflammation limited factor in proinflammatory phenotype cells inhibiting succinate dehydrogenase and causing increases of succinate and citrate (29-31). Anti-inflammatory phenotype cells (i.e., M2 macrophages, NKT cells and Treg cells) are associated with increased OXPHOS and decreased succinate and citrate (32-35). MECs involve in the occurrence and development of $S$. uberis-induced inflammation in our previous study $(13,15)$. EpH4-Ev cells incubated with $S$. uberis for $3 \mathrm{~h}$ have decreased citrate and a-ketoglutarate but increased itaconate levels (Fig. 2A and Fig. S6A). We postulated that different metabolic patterns were present in MECs and proinflammatory phenotype innate immune cells challenged by $S$. uberis. Inactivated $S$. uberis, Escherichia coli (E. coli) and lipopolysaccharide (LPS) were used to stimulate EpH4-Ev cells and RAW 264.7 macrophages. LPS-stimulated RAW 264.7 macrophages developed an increase in extracellular acidification rate (ECAR; reflects glycolysis rate) and a decrease in oxygen consumption rate (OCR; reflects mitochondrial function and OXPHOS level) levels. MECs had an increase in ECAR and OCR levels after LPS challenge (Fig. 3A-D). Both ECAR and OCR levels increased in the above 2 cell lines with inactivated E. coli and S. uberis stimulation (Fig. 3A-D). These data indicate that MECs adopt distinct metabolism in response to various microbial stimuli compared to macrophages.

\section{Metabolic reprogramming by taurine in S. uberis infection coordinates with the energy supply and production of anabolic intermediates}

Cell metabolism generates energy and intermediates used to synthesize materials required to combat pathogen infection (36). Excessive or disordered mobilization results in cellular dysfunction. We evaluated whether taurine regulated metabolism associated with energy supply and anabolic intermediates production during S. uberis infection. Taurine pretreatment lowered ECAR and OCR levels during $S$. uberis challenge indicating that glycolysis and OXPHOS were inhibited in S. uberis-infected MECs (Fig. 4A-B). Cells obtain energy and anabolic intermediates via biochemical reactive catalytic 
enzymes. Homeostasis is critical to cellular function and stability. We found that the activities of hexokinase (HK; the first and rate-limiting enzyme of glycolysis), phosphofructokinase (PFK; the ratelimiting enzyme), and lactate dehydrogenase (LDH) increased in EpH4-Ev cells challenged with $S$. uberis (Fig. 4C-E). The activities of pyruvate dehydrogenase (PDH), succinate dehydrogenase (SDH), and mitochondrial complex IV (key enzymes or components in OXPHOS) also elevated (Fig. 4F-H). Taurine pretreatment significantly decreased these levels in infected EpH4-Ev cells (Fig. 4C-H). These results agreed with real-time measurements of ECAR and OCR. Thus, MECs exhibit enhanced glycolysis and OXPHOS in response to $S$. uberis infection which are attenuated by taurine pretreatment.

To investigate whether $S$. uberis challenge promoted the conversion between amino acid and energyrelated metabolites (such as glutamate and a-ketoglutarate) associated with a decrease in TCA intermediates, we assayed the activities of several key enzymes involved in energy and amino acid metabolism. S. uberis infection significantly increased glutamate dehydrogenase (GDH) (Fig. 4I), glutamic oxalacetic transaminase (GOT) (Fig. 4J), and glutamic-pyruvic transaminase (GPT) activity (Fig. 4K). Taurine pretreatment attenuated these changes. These results indicate that taurine reprograms S. uberis-induced metabolic changes to coordinate the energy supply and anabolic intermediate production.

\section{Taurine balance metabolism to alleviate inflammation induced by $S$. uberis}

S. uberis promotes pro-inflammatory mediator production in mammary glands and MECs $(12,15)$. EpH4Ev cells were treated with 2-deoxy-D-glucose (2-DG; which inhibits glycolysis) and CPI-613 (which inhibits pyruvate dehydrogenase and a-ketoglutarate dehydrogenase, thereby blocking OXPHOS) to explore the relationship between energy metabolism and the inflammatory response. Key enzymes of glycolysis and OXPHOS detection showed that these 2 carbohydrate pathways were significantly restricted in EpH4-Ev cells with 2-DG (Fig. S7A-C) and CPI-613 (Fig. S7D-F), respectively. The effects of taurine pretreatment on glycolysis and OXPHOS were similar to those observed with 2-DG or CIP-613 treatment.

Taurine pretreatment significantly decreased TNF-a and IL-1 $\beta$ levels in EpH4-Ev cells infected with $S$. uberis (Fig. 5A-B), which was comparable to that of 2-DG treatment. Similarly, pretreatment of S. uberis infected EpH4-Ev cells with both 2-DG and taurine downregulated intracellular ROS levels more than pretreatment with taurine alone (Fig. 5C). The supernatants of EpH4-Ev cells pretreated with taurine or glycolysis inhibitors and later infected with $S$. uberis had lower activity of NAGase (a marker of cell damage) than that of $S$. uberis-infected EpH4-Ev cells without pretreatment (Fig. 5D). OXPHOS inhibition significantly increased TNF- $\alpha$ and IL-1 $\beta$ production (Fig. 5E-F), contrary to taurine pretreatment. Moreover, CPI-613 treatment significantly increased intracellular ROS levels (Fig. 5G) and NAGase activities (Fig. 5H) in EpH4-Ev cells during S. uberis infection. Inhibited glycolysis with 2-DG diminished the inflammatory response while CPI-613 treatment results in a sharp increase of inflammatory mediators and resultant cell damage in $S$. uberis infection. These results suggest that glycolysis and OXPHOS work differently in MECs challenged with S. uberis. Taurine decreases both glycolysis and OXPHOS balancing 
whole energy metabolism in S. uberis infection, relieving inflammation and protecting cells from OXPHOS breakdown thus attenuating a proinflammatory metabolic reaction.

\section{Taurine regulates metabolic alterations in S. uberis infection by inhibiting the mTOR pathway}

Previously, we show that mTOR was involved in the production of various inflammatory mediators during S. uberis infection (15). Enhancement of the glycolytic pathway correlates with mTOR activity (36). Therefore, we hypothesized that taurine coupled with cellular metabolism through the mTOR pathway optimizes the production of inflammatory biomolecules. Taurine pretreatment inhibited mTOR pathway activity in EpH4-Ev cells during challenge with $S$. uberis, as indicated by decreased mTOR phosphorylation and decreased phosphorylation of its downstream targets, p70 S6K and 4E-BP1 (Fig. 6A). The mTOR activator, MHY1485, reversed these changes in taurine-pretreated EpH4-Ev cells (Fig. 6A). To verify whether mTOR mediated energy metabolism during $S$. uberis infection, we assessed ECAR and OCR levels using MHY1485. ECAR and OCR decreased in taurine-pretreated EpH4-Ev cells exposed to S. uberis (Fig. 6B-C), whereas MHY1485 treatment increased these levels (Fig. 6B-C). The activities of key glycolysis-associated (HK, PFK, LDH) and OXPHOS-associated (PDH, SDH, mitochondrial complex IV) enzymes or components were consistent with the changes of ECAR and OCR (Fig. 6D-I). These findings confirm that taurine-mediated attenuation of metabolic responses in EpH4-Ev cells challenged with $S$. uberis is related to mTOR pathway activation. Additionally, TNF-a and IL-1 $\beta$ levels (Fig. 6J-K), ROS production (Fig. 6L and Fig. S8), and NAGase activity (Fig. 6M) significantly decreased in taurine-pretreated EpH4-Ev cells during S. uberis infection, whereas MHY1485 treatment exerted the opposite effects of taurine. These data establish that the mTOR signaling pathway mediates metabolic alterations of taurine in $S$. uberis infection.

\section{Taurine rescues mTOR-mediated metabolic alterations in MECs via AMPK}

As an AMPK activator (Fig. S9A), taurine has potential to negatively regulate mTOR activity resulting in coordination of cell metabolism with specific energy requirements (27). In EpH4-Ev cells, S. uberis infection increased the levels of phosphorylated AMPK versus controls (Fig. 7A). To determine whether the AMPK pathway was involved in the metabolic recovery induced by taurine, we assessed the expression status of the AMPK-mTOR-p70 S6K-4E-BP1 pathway in MECs after taurine pretreatment. Immunoblot analyses showed that taurine pretreatment significantly increased AMPK phosphorylation levels and subsequently downregulated mTOR, p70 S6K, and 4E-BP1 phosphorylation all of which function immediately downstream of mTOR (Fig. 7A). Pretreatment with the AMPK inhibitor, Compound $\mathrm{C}$, blocked taurine-induced AMPK phosphorylation and reversed mTOR, p70 S6K, and 4E-BP1 phosphorylation (Fig. 7A). ECAR and OCR levels increased, which concurred with the activity level of the mTOR pathway (Fig. 7B-C). These data suggest that taurine-mediated regulation of metabolism in $S$. uberis-infected EpH4-Ev cells occurs via AMPK. Taurine significantly decreased HK, PFK, LDH, PDH, SDH, and mitochondrial complex IV activities in S. uberis infection, while pretreatment with Compound C reversed these taurine effects (Fig. 7D-I). Taurine significantly decreased the levels of TNF- $a$ and IL-1 $\beta$ (Fig. 7J-K), ROS production (Fig. 7L and Fig. S9B), and NAGase activity (Fig. 7M) in S. uberis-infected EpH4-Ev cells, whereas Compound C pretreatment reversed these taurine-induced effects. 


\section{Discussion}

Propagation of the intracellular bacterial pathogen S. uberis is strictly bound to its host cells. Many of the vital nutrients are synthesized only by the host, and this has complex implications. S. uberis actively interact with the host, modulating host metabolism and stimulating the immune response, which in turn affects the physiology of both, the host cell and the bacteria. Host metabolism homeostasis contributes significantly to combat invaders. However, the relative impact of metabolism pathways versus cellular inflammation on the progression of $S$. uberis infection remains poorly understood.

Mastitis remains an ongoing health problem, especially when caused by persistent intracellular bacterial infection, such as $S$. uberis $(1,7)$. Recent findings in our lab have shown that inflammation is tightly controlled, which protects the host from excessive or chronic damage induced by this process (13). We have found that taurine plays an important role in $S$. uberis infection for protecting host from inflammation over years (11-13). As metabolism and inflammation are closely interconnected, we previously investigated whether taurine ameliorates $S$. uberis induced mastitis via metabolic alteration. We employed GC-TOF-MS non-targeted metabolomics methodology to analyze the metabolic changes in mammary glands and MECs after $S$. uberis infection. The resultant data demonstrate that during $S$. uberis invasion, the intermediates of glycolysis, the pentose phosphate pathway, lipid metabolism and amino acid metabolism are significantly increased while the concentration of TCA cycle intermediates decrease. In addition, taurine has a regulatory effect on these changes, which suggests that its regulation of $S$. uberis infection is related to metabolism. The metabonomic data presented here show that the alteration of metabolic pattern in MECs are not completely matched with those in mammary tissue. Mammary glands are complex and contain many cell types besides MECs partially obfuscating in vivo comparisons.

MECs are the major functional cells of the mammary gland, and although they are not professional immune cells, they are involved in inflammatory responses to $S$. uberis infection $(13,15)$. The role of MECs in metabolic changes occurring in the mammary gland, and the significance of these metabolic changes are not known. It has been reported that metabolic changes impact cell functions (37), and metabolic reprogramming is associated with an adjustment of host defense ability (38). For example, during activation, immune cells, such as macrophages, monocytes, and DCs, modulate their metabolism by increasing glycolytic fluxes enhancing phagocytic capacity or secretion of immunoregulatory cytokines (32). Human monocytes stimulated with LPS shift from OXPHOS to glycolysis, thereby activating host defense factors production (39). In the current study, the levels of glycolysis and OXPHOS in MECs increased during $S$. uberis infection. By means of these metabolic alerts, the increased energy and intermediary requirements were met for defensive host immune responses (40). This indicates that metabolic reprogramming of MECs plays a crucial role in mammary defense.

Different from typical proinflammatory phenotypes, immune cells (M1 macrophages, NKs and PMN, etc.) and even intestinal epithelial cells $(33,41-43)$, MECs undergo a unique metabolic reprogramming pattern characterized by decreased production of TCA cycle intermediates accompanied by activated OXPHOS. 
This was further confirmed by the activities of key enzymes in glycometabolism. Specific metabolic alterations in cells accommodate distinct functional outputs. Glycolysis upregulation is more likely to occur under inflammatory conditions, whereas OXPHOS represents upregulation of anti-inflammatory pathways $(35,44-46)$. Increased activity of the glycolytic pathway likely promotes inflammation and resistance to $S$. uberis infection, although the precise reason for OXPHOS is also upregulated in $S$. uberisinfected MECs remains unclear. A likely explanation is that MECs act as part-time immune cells and are, therefore, not equipped to counter the acute challenge during $S$. uberis infection. Therefore, they may enhance OXPHOS by producing more energy and contributing more intermediates to constrain infection and subsequent inflammation, thereby reducing cell damage (40). Our results show that glycolysis inhibition in MECs through 2-DG reduced ROS, TNF-a, and IL-1 $\beta$ secretion alleviates inflammation and subsequent injury. Blocking OXPHOS through CPI-613 leads to increased inflammation. The unique metabolic shift in MECs may represent a specific self-protective mechanism that relieves excessive inflammation during $S$. uberis infection.

In mammalian cells, taurine is derived from cysteine and has many biological roles, such as conjugation of bile acids (absorption and transport of lipids), osmoregulation, and regulation of glucose and lipid homeostasis (energy metabolism) $(19,47)$. In diabetic rats, taurine supplementation reduces abdominal body fat while improving glucose tolerance (48). In the current in vitro metabonomic study, taurine reduces the activities of several key glycolytic enzymes, the TCA cycle and amino-acid metabolism, suggesting that taurine alleviates excessively mobilized substances and affects energy metabolism in $S$. uberis activated MECs.

The AMPK-mTOR pathway functions as a signaling nexus for regulating cellular metabolism, energy homeostasis, and cell growth under various nutrient stress. In most cells, AMPK plays an important role in sensing energy status and restoring energy balance (27). We find taurine is an AMPK activator in this study. AMPK activators exert anti-inflammatory effects by directly activating catabolism and inhibiting anabolism (35). Our data show that taurine constrained both glycolysis and OXPHOS, indicating that it may regulate overall energy production in S. uberis infected MECs. We conclude that AMPK mediates metabolism regulation in S. uberis infected MECs. mTOR is a central controller of cell growth and proliferation through its influence on metabolism, especially on protein synthesis. AMPK is a master regulator of metabolism that acts upstream of mTOR. Its negative control of the mTOR pathway correlates with glycolytic activity. In macrophages, mTOR-mediated proinflammatory signal stimulation can drive mTOR-dependent metabolic-flux reprogramming (36). Additionally, LPS-treated macrophages display increased inflammatory responses through PI3K/mTOR pathway activation (49). These data are consistent with our previous finding that $\mathrm{PI} 3 \mathrm{~K} / \mathrm{Akt} / \mathrm{mTOR}$-pathway activation promotes inflammation in S. uberis-infected MECs (15). It is therefore possible that taurine regulates metabolism through the AMPKmTOR pathway thus reducing inflammation.

In the current study, taurine alleviates $S$. uberis induced-inflammation by activating AMPK which inhibits the mTOR pathway, resulting in decreased proinflammatory cytokine production and decreased anabolic and catabolic processes. Treatment with AMPK inhibitors or MTOR agonists considerably increases the 
expression of inflammatory factors and ROS, causing further cell damage. Compound C, an AMPK inhibitor, reverses taurine-induced AMPK phosphorylation and altered mTOR activity thus regulating the corresponding metabolic changes and anti-inflammatory responses. Taken together, our results show that taurine decreases overall energy metabolism in the host by regulating the AMPK-mTOR pathway, reducing inflammation caused by $S$. uberis infection.

Our findings show that metabolic reprogramming drives $S$. uberis-induced inflammation in mammary glands and MECs, and that taurine abrogates this metabolic reprogramming and dysfunction, thereby reducing inflammation. Taurine effectively inhibits overall energy metabolism by boosting AMPK levels reducing the activity of the mTOR pathway, which markedly reduces $S$. uberis-induced inflammatory responses in MECs. Our findings shed light on the close relationship between cellular-metabolic pathways and inflammation of mammary glands and MECs.

\section{Materials And Methods}

\section{Key resources table}




\begin{tabular}{|c|c|c|}
\hline Reagent or Resource & SOURCE & IDENTIFIER \\
\hline \multicolumn{3}{|l|}{ Antibodies } \\
\hline AMPKa antibody & Cell Signaling Technology, Boston, USA & Cat. \#2532 \\
\hline $\begin{array}{l}\text { Phospho-AMPK (Thr172) (40H9) } \\
\text { Rabbit mAb }\end{array}$ & Cell Signaling Technology, Boston, USA & Cat. \#2535 \\
\hline mTOR antibody & Cell Signaling Technology, Boston, USA & Cat. \#2972S \\
\hline $\begin{array}{l}\text { Phospho-mTOR (Ser2448) } \\
\text { (D9C2) XP Rabbit mAb }\end{array}$ & Cell Signaling Technology, Boston, USA & Cat. \#5536 \\
\hline $\begin{array}{l}\text { p70 S6 Kinase (49D7) Rabbit } \\
\text { mAb }\end{array}$ & Cell Signaling Technology, Boston, USA & Cat. \#2708 \\
\hline $\begin{array}{l}\text { Phospho-p70 S6 Kinase } \\
\text { (Thr389) antibody }\end{array}$ & Cell Signaling Technology, Boston, USA & Cat. \#9205 \\
\hline 4E-BP1(53H11) Rabbit mAb & Cell Signaling Technology, Boston, USA & Cat. \#9644 \\
\hline $\begin{array}{l}\text { Phospho-4E-BP1 (Thr37/46) } \\
\text { (236B4) Rabbit mAb }\end{array}$ & Cell Signaling Technology, Boston, USA & Cat. \#2855 \\
\hline $\begin{array}{l}\text { B-Actin (4D3) monoclonal } \\
\text { antibody }\end{array}$ & Bioworld, Nanjing, China & Cat. \#BS6007M \\
\hline $\begin{array}{l}\text { Anti-rabbit lgG, HRP-linked } \\
\text { Antibody }\end{array}$ & Cell Signaling Technology, Boston, USA & Cat. \#7074S \\
\hline \multicolumn{3}{|l|}{ Chemicals } \\
\hline Taurine & Sangon Biotech, Shanghai, China & $\begin{array}{l}\text { Cat. \#a500926-0100; } \\
\text { CAS: } 107-35-7\end{array}$ \\
\hline $\begin{array}{l}\text { Lipopolysaccharides (LPS) } \\
\text { fromEscherichia coli055: B5 }\end{array}$ & Solarbio, Beijing, China & Cat. \#L8880 \\
\hline \multirow[t]{2}{*}{ 2-Deoxy-D-glucose } & Sigma-Aldrich, USA & Cat. \#D8375; \\
\hline & & CAS: $154-17-6$ \\
\hline \multirow[t]{2}{*}{ CPI-613, Devimistat } & Selleck Chemicals, USA & Cat. \# S2776; \\
\hline & & CAS: $95809-78-2$ \\
\hline \multirow{2}{*}{$\begin{array}{l}\text { Dorsomorphin (Compound C) } \\
2 \mathrm{HCl}\end{array}$} & Selleck Chemicals, USA & Cat. \#S7306; \\
\hline & & CAS: $1219168-18-9$ \\
\hline \multirow[t]{2}{*}{ MHY1485 } & Selleck Chemicals, USA & Cat. \#S7811; \\
\hline & & CAS: 326914-06-1 \\
\hline \multicolumn{3}{|l|}{ Commercial Assays } \\
\hline Mouse TNF- $a$ ELISA kit & Rigor Bioscience, Beijing, China & Cat. \#RGB-60080M \\
\hline
\end{tabular}




\begin{tabular}{|c|c|c|}
\hline Reagent or Resource & SOURCE & IDENTIFIER \\
\hline Mouse IL-1 $\beta$ ELISA kit & Rigor Bioscience, Beijing, China & Cat. \#RGB-60013M \\
\hline Hexokinase (HK) Assay Kit & Solarbio, Beijing, China & Cat. \#BC0745 \\
\hline $\begin{array}{l}\text { Phosphofructokinase (PFK) } \\
\text { Assay Kit }\end{array}$ & Solarbio, Beijing, China & Cat. \#BC0535 \\
\hline $\begin{array}{l}\text { Lactic dehydrogenase (LDH) } \\
\text { Assay Kit }\end{array}$ & Solarbio, Beijing, China & Cat. \#BC0685 \\
\hline $\begin{array}{l}\text { Pyruvate dehydrogenase (PDH) } \\
\text { Assay Kit }\end{array}$ & Solarbio, Beijing, China & Cat. \#BC0380 \\
\hline $\begin{array}{l}\text { Succinodehydrogenase (SDH) } \\
\text { Assay Kit }\end{array}$ & Solarbio, Beijing, China & Cat. \#BC0950 \\
\hline $\begin{array}{l}\text { Mitochondrial Complex IV Assay } \\
\text { Kit }\end{array}$ & Solarbio, Beijing, China & Cat. \#BC0945 \\
\hline $\begin{array}{l}\text { N-acetyl- } \beta-D-\text {-Glucosaminidase } \\
\text { (NAGase) Assay Kit }\end{array}$ & $\begin{array}{l}\text { Jiancheng, Bioengineering Institute, } \\
\text { Nanjing, China }\end{array}$ & Cat. \#A031 \\
\hline $\begin{array}{l}\text { Reactive Oxygen Species (ROS) } \\
\text { Assay Kit }\end{array}$ & Beyotime, Nantong, China & Cat. \#S0033 \\
\hline Seahorse ECAR Assay Kit & Seahorse Biosciences, USA & N/A \\
\hline Seahorse OCR Assay Kit & Seahorse Biosciences, USA & N/A \\
\hline $\begin{array}{l}\text { Chemiluminescence Kit (Super } \\
\text { ECL Reagent substrate) }\end{array}$ & Hai Gene, Harbin, China & Cat. \#M2301 \\
\hline \multicolumn{3}{|c|}{ Experimental Models: Bacterial Strains } \\
\hline Streptococcus uberis $0140 \mathrm{~J}$ & ATCC, Manassas, USA & Cat. \#ATCCBAA-854 \\
\hline Escherichia ColiNJ-17 & $\begin{array}{l}\text { Academy of Agricultural Sciences, } \\
\text { Jiangsu, China }\end{array}$ & N/A \\
\hline \multicolumn{3}{|l|}{ Experimental Models: Cell Lines } \\
\hline $\begin{array}{l}\text { EpH4-EvMus musculusbreast } \\
\text { epithelial cell line }\end{array}$ & ATCC, Manassas, USA & $\begin{array}{l}\text { Cat. \#ATCCCRL- } \\
3063\end{array}$ \\
\hline RAW264.7 macrophage cell line & ATCC, Manassas, USA & Cat. \#ATCCTIB-71 \\
\hline \multicolumn{3}{|c|}{ Experimental Models: Organisms/Strains } \\
\hline C57BL/6J mice & $\begin{array}{l}\text { Bio Medical Research Institute of } \\
\text { Nanjing University, Nanjing, China }\end{array}$ & N/A \\
\hline \multicolumn{3}{|l|}{ Software and Algorithms } \\
\hline GraphPad Prism v7 & GraphPad Software & N/A \\
\hline ImageJ v.2.0.0 & $\mathrm{NIH}$ & $\mathrm{N} / \mathrm{A}$ \\
\hline
\end{tabular}




\begin{tabular}{|lll|}
\hline Reagent or Resource & SOURCE & IDENTIFIER \\
\hline FlowJo & Tree Star & N/A \\
\hline Other & & Cat. \#CM0189 \\
\hline Todd Hewitt Broth & Sigma-Aldrich, USA & Cat. \#11965-065 \\
\hline DMEM(1X) & Gibco, New York, USA & Cat. \#25200-056 \\
\hline $\mathbf{0 . 2 5 \%}$ Trypsin-EDTA (1X) & Gibco, New York, USA & Cat. \#10099-141 \\
\hline $\begin{array}{l}\text { Fetal Bovine Serum } \\
\begin{array}{l}\text { Polyvinylidene fluoride } \\
\text { membrane }\end{array}\end{array}$ & Gibco, New York, USA & N/A \\
\hline
\end{tabular}

\section{Bacterial strains and growth conditions}

S. uberis (0140J strain) was inoculated into Todd-Hewitt broth medium containing $2 \%$ fetal bovine serum (FBS, Gibco, USA) and cultured at $37^{\circ} \mathrm{C}$ in an orbital shaker to log-phase growth $\left(\mathrm{OD}_{600}=0.4-0.6\right)$. E. coli (NJ-17 strain) was inoculated into Luria-Bertani medium at $37^{\circ} \mathrm{C}$ and cultured in an orbital shaker to log-phase growth $\left(\mathrm{OD}_{600}=0.5-0.6\right)$.

\section{Mouse infections}

Female C57BL/6J mice (6-8 weeks old) were used for the animal experiments.

All procedures involving animals were approved by the committee on the Use and Care of Animals of Nanjing Agricultural University (Nanjing, China).

Our protocol number approved by the Animal welfare committee is PZ2019144.

Twenty-four healthy pregnant mice were housed in individual cages and provided water and food ad libitum. Following acclimatization, the mice were randomly divided into 4 groups (Control, Taurine, $S$. uberis, and Taurine $+S$. uberis groups); each group contained 6 mice. Starting on gestation day 14,100 $\mathrm{mg} / \mathrm{kg}$ taurine (dissolved in sterile pyrogen-free saline), or an equal volume of saline, was administered daily to each mouse by gavage until parturition. At $72 \mathrm{~h}$ after parturition, mice in the $S$. uberis and Taurine $+S$. uberis groups were infused with 100 CFUs S. uberis in $50 \mu \mathrm{L}$ into the $\mathrm{L} 4$ and R4 teats. The offspring were weaned $2 \mathrm{~h}$ prior to this infusion. Following administration of ether anesthesia, the L4 and R4 teats were moistened with $75 \%$ ethanol, and a 33-gauge needle (fitted to a 1-mL syringe) was gently inserted into the mammary duct, after which $50 \mu \mathrm{L} \mathrm{S}$. uberis was slowly infused. At $24 \mathrm{~h} \mathrm{Pl}$, all mice in the 4 groups were euthanized, and the mammary glands were aseptically collected and stored at $-80^{\circ} \mathrm{C}$ until analysis.

\section{EpH4-Ev cell culture and treatment}

EpH4-Ev cells were grown at $37^{\circ} \mathrm{C}$ in DMEM supplemented with $10 \% \mathrm{FBS}$ in 6-well plates until they reached $70-80 \%$ confluency. The resulting monolayers were cultured in FBS-free DMEM for $4 \mathrm{~h}$ and then 
further cultured in FBS-free DMEM for $24 \mathrm{~h}$, with or without $45 \mathrm{mmol} / \mathrm{L}$ taurine. Next, both groups of cells were infected with $S$. uberis at an MOI of 10 for 1,2 , or $3 \mathrm{~h}$.

Cell samples prepared for GC-TOF-MS analysis were collected as described previously (50). Briefly, cells (plated at approximately $5 \times 10^{6}$ cells/well) were infected with $S$. uberis for 1,2 , or $3 \mathrm{~h}$. The cell supernatants were discarded, and the cells were washed three times with cold phosphate-buffered saline (PBS), quenched with $400 \mu \mathrm{L}$ cold methanol (precooled at $-80^{\circ} \mathrm{C}$ ), and held at $-80^{\circ} \mathrm{C}$ for $30 \mathrm{~min}$. An additional $400 \mu \mathrm{L}$ double distilled water was added to the plates, and the cells were scraped off separate plates. The cell suspensions were stored at $-80^{\circ} \mathrm{C}$ and then used to prepare samples for metabolomics analysis.

For extracellular flux analysis, EpH4-Ev cells were challenged with $10 \mu \mathrm{g} / \mathrm{mL}$ LPS for $12 \mathrm{~h}$, or with inactivated S. uberis or E. coli NJ-17 at a $\mathrm{MOI}$ of 100 for $3 \mathrm{~h}$, at $37^{\circ} \mathrm{C}$.

For the inhibition and activation experiments, cells were pretreated as described below before $S$. uberis infection. Specifically, EpH4-Ev cells were grown at $37^{\circ} \mathrm{C}$ in DMEM with $10 \% \mathrm{FBS}$ in 6 -well plates and grown to $70-80 \%$ confluency. After culturing the cells in FBS-free DMEM for $4 \mathrm{~h}$, the monolayers were treated with $5 \mathrm{mM}$ 2-DG (Selleckchem) for $1 \mathrm{~h}, 25 \mu \mathrm{M}$ CPI-613 (Selleckchem) for $12 \mathrm{~h}, 100 \mathrm{nM}$ MHY1485 (Selleckchem) for $24 \mathrm{~h}$, or $10 \mu \mathrm{M}$ Compound C (Selleckchem) for $1 \mathrm{~h}$. All the inhibitors or activators used in this study had been determined by cell viability and they were nonpoisonous to cells.

\section{Culturing and treating RAW 264.7 macrophages}

For extracellular flux analysis, RAW 264.7 macrophages were grown to $70-80 \%$ confluency in DMEM containing $10 \%$ FBS in XF 24-well plates. Next, the cells were stimulated with $100 \mathrm{ng} / \mathrm{mL}$ LPS for $12 \mathrm{~h}$ or with inactivated S. uberis or E. coli NJ-17 at an MOI of 5 for 3 h, at $37^{\circ} \mathrm{C}$.

\section{Metabolite extraction for GC-TOF-MS}

A $50 \pm 1 \mathrm{mg}$ sample of each mammary gland was extracted with $450 \mu \mathrm{L}$ extraction buffer $\left(\mathrm{V}_{\text {Methanol: }}\right.$ : $\left.\mathrm{V}_{\text {Chloroform }}=3: 1\right)$ and placed into 2-mL EP tubes. Next, $10 \mu \mathrm{L} \mathrm{I-2-chlorophenylalanine}(1 \mathrm{mg} / \mathrm{mL}$ stock in $\mathrm{dH}_{2} \mathrm{O}$ ) was added to each mammary gland sample and vortexed for $30 \mathrm{~s}$. Subsequently, $10 \mu \mathrm{L}$ adonitol $\left(0.5 \mathrm{mg} / \mathrm{mL}\right.$ stock in $\left.\mathrm{dH}_{2} \mathrm{O}\right)$ was added to the cell samples, followed by vortexing again for $30 \mathrm{~s}$. The vortexed samples were homogenized for $4 \mathrm{~min}$ then ultrasonicated for 5 min while maintained in an icewater bath. The ultrasonicated samples were centrifuged for $15 \mathrm{~min}$ at $5,000 \times g$ at $4^{\circ} \mathrm{C}$. Next, approximately $350 \mu \mathrm{L}$ supernatant from the mammary-gland samples, or $600 \mu \mathrm{L}$ supernatant from the cell samples, was transferred into fresh $1.5 \mathrm{~mL}$ EP tubes; $40 \mu \mathrm{L}$ supernatant from each sample was pooled for quality control. The samples were dried completely in a vacuum concentrator without heating, and each mammary gland sample or cell sample was resuspended in 60 or $20 \mu \mathrm{L}$ methoxyamine hydrochloride $\left(20 \mathrm{mg} / \mathrm{mL}\right.$ in pyridine), respectively. After incubation for $30 \mathrm{~min}$ at $80^{\circ} \mathrm{C}$, approximately 80 $\mu \mathrm{L} \mathrm{N}, \mathrm{O}$-Bis (trimethylsilyl) trifluoroacetamide (BSTFA) reagent (1\% trimethylchlorosilane (TMCS), $\mathrm{v} / \mathrm{v}$ ) was added to the sample aliquots and incubated for $1.5 \mathrm{~h}$ at $70^{\circ} \mathrm{C}$ before GC-TOF-MS analysis. 


\section{GC-TOF-MS analysis}

GC-TOF-MS analyses were performed using an Agilent $7890 \mathrm{GC}$ instrument coupled with a Pegasus 4D TOF-MS instrument. This system utilized a DB-5MS capillary column coated with $5 \%$ diphenyl crosslinked with 95\% dimethylpolysiloxane (30 $\mathrm{m} \times 250 \mu \mathrm{m}$ [inner diameter], 0.25- $\mu \mathrm{m}$ film thickness; J\&W Scientific, Folsom, CA, USA). Next, 1- $\mu \mathrm{L}$ aliquots of the analytes were injected in splitless mode. Helium was used as the carrier gas, the front inlet purge flow was $3 \mathrm{~mL} / \mathrm{min}$, and the gas flow rate through the column was $1 \mathrm{~mL} / \mathrm{min}$. The initial temperature was maintained at $80^{\circ} \mathrm{C}$ for $1 \mathrm{~min}$, raised to $290^{\circ} \mathrm{C}$ at a rate of $10^{\circ} \mathrm{C} / \mathrm{min}$, and then maintained for $8 \mathrm{~min}$ at $290^{\circ} \mathrm{C}$. The injection, transfer line, and ion-source temperatures were 280,295 , and $220^{\circ} \mathrm{C}$, respectively. Ionization was performed at $-70 \mathrm{eV}$ in electronimpact mode. MS data were acquired in full-scan mode with a mass/charge range of 50 to 600 , at a rate of $10 \mathrm{spectra/s}$ after a solvent delay of $7.9 \mathrm{~min}$.

\section{Detection of relative enzyme activities}

The HK, PFK, LDH, PDH, SDH, mitochondrial complex IV, and NAGase activities in EpH4-Ev cells was measured using commercial kits according to the instructions of respective manufacturers.

\section{Extracellular flux analysis}

Real-time OCR and ECAR values of EpH4-Ev cells or RAW 264.7 cells were analyzed using an XF-24 Extracellular Flux Analyzer (Seahorse Bioscience, Boston, MA, USA). Briefly, the basal metabolic rates of cells seeded in quintuplicate were determined using 4 consecutive measurements in unbuffered, sterilefiltered Seahorse medium (8.3 g DMEM powder, $0.016 \mathrm{~g}$ phenol red, and $1.85 \mathrm{~g} \mathrm{NaCl}$ in $1 \mathrm{~L}$ Milli-Q water, $\mathrm{pH}=7.4$ at $37^{\circ} \mathrm{C}$ ) containing $2 \mathrm{mM}$ glutamine (for ECAR measurements) or $10 \mathrm{mM}$ glucose, $2 \mathrm{mM}$ glutamine, and $1 \mathrm{mM}$ sodium pyruvate (for OCR measurements). The cells were incubated in a $\mathrm{CO}_{2}$-free incubator at $37^{\circ} \mathrm{C}$ for $1 \mathrm{~h}$ before measurements. After 3 basal measurements, 3 consecutive measurements were acquired after the different treatments. For ECAR detection, the cells were sequentially exposed to $10 \mathrm{mM}$ glucose, $2 \mathrm{mM}$ oligomycin, and $50 \mathrm{mM}$ 2-DG. For OCR determinations, the cells were sequentially exposed to $2 \mathrm{mM}$ oligomycin, $1 \mathrm{mM} \mathrm{FCCP}$, and $2 \mu \mathrm{M}$ antimycin $\mathrm{A}+1 \mu \mathrm{M}$ rotenone. Next, $20 \mathrm{mM}$ glucose and $1 \mathrm{mM}$ pyruvate were added along with FCCP to fuel maximal respiration. All compounds used during the Seahorse runs were acquired from Sigma-Aldrich. The cell number in each well was normalized by measuring the total protein content, using a Synergy ${ }^{\mathrm{TM}} \mathrm{H} 1 \mathrm{Hybrid}$ Multi-Mode Microplate Reader (BioTek Instruments). The 24-well microplates used for Seahorse measurements were pretreated with Cell-Tak Cell and Tissue Adhesive (Corning).

\section{ELISA testing}

TNF- $\alpha$ and IL-1 $\beta$ levels in EpH4-Ev cells were measured using ELISA kits ((Rigor Bioscience, Beijing, China), according to the manufacturer's instructions.

\section{ROS measurements}

Per the manufacturer's instructions of the ROS assay kit (Beyotime, Nantong, China), EpH4-Ev cells were incubated in $10 \mu \mathrm{mol} / \mathrm{L} \mathrm{DCFH-DA}$ for $30 \mathrm{~min}$ at $37^{\circ} \mathrm{C}$, washed 3 times in PBS, and detached with trypsin. The cells were centrifuged at $400 \times g$ for $5 \mathrm{~min}$, resuspended in PBS, and immediately analyzed by flow 
cytometry using a FACSCanto instrument; 10,000 cells/ sample were analyzed using CellQuest Pro acquisition software and FlowJo software.

\section{Western blotting}

Intracellular protein levels were determined by western blotting. An anti-b-actin antibody (Bioworld, USA) was used as a loading control. Cells were washed twice in ice-cold PBS and lysed by incubation in RIPA buffer (Beyotime, Nantong, China) containing PMSF (Beyotime, Nantong, China) on ice for 30 min. Supernatants were collected by centrifuging at $5000 \times g$ for $10 \mathrm{~min}$ at $4^{\circ} \mathrm{C}$, and protein concentrations were determined using a Bicinchoninic Acid Assay Kit (Beyotime, Nantong, China) and detected with a spectrophotometer (Tecan, Männedorf, Switzerland). Proteins were separated by electrophoresis on a polyacrylamide gel and transferred to polyvinylidene fluoride membranes (Millipore, USA). The membranes were blocked with 5\% non-fat milk diluted in Tris buffered saline with Tween-20 (TBST) for 2 $\mathrm{h}$ at room temperature (approximately $10-25^{\circ} \mathrm{C}$ ) and hybridized overnight with an appropriate primary antibody at $4^{\circ} \mathrm{C}$. Primary antibodies were diluted in TBST as follows: $\beta$-actin (1: 10,000), AMPKa (1: 1,000), p-AMPK (1: 1,000), mTOR (1: 1,000), p-mTOR (1: 1,000), p70 S6K (1: 1,000), p-p70 S6K (1: 1,000), 4E-BP1 (1: 1,000), and p-4E-BP1 (1: 1,000). The membranes were washed three times with TBST before and after incubation with a horseradish peroxidase (HRP)-linked anti-rabbit IgG (CST, Massachusetts, USA, $1: 10,000$ ) secondary antibody at room temperature (approximately $10-25^{\circ} \mathrm{C}$ ) for $2 \mathrm{~h}$. Signals were detected using an ECL Western Blot Analysis System (Tanon, Shanghai, China). Bands were quantified using ImageJ software (NIH, USA).

\section{Statistical analysis}

Statistical analysis was performed using GraphPad Prism 7 software. All data are represented as the mean \pm SEM. To meet the assumption of homogeneity of variance, an analysis of variance (ANOVA) was performed, followed by Tukey's multiple comparison test. The Student's t-test was used to compare the difference between two groups, and differences were considered statistically significant at $p<0.05$.

\section{Declarations}

\section{ACKNOWLEDGMENTS}

This project was supported by grants from the National Natural Science Foundation of China (No. 32072867, 31672515), the Key Project of Inter-governmental International Scientific and Technological Innovation Cooperation (No.2018YFE0102200), and the Project Funded by the Priority Academic Program Development of Jiangsu Higher Education Institutions. We thank Dr. Howard Gelberg (Oregon State University) for editing this manuscript.

\section{AUTHOR CONTRIBUTIONS}

R.G.L. and Z.X.W. contributed equally as co-first authors. R.G.L. and Z.X.W. designed and performed the experiments and analyzed the data. Z.X.W. wrote the manuscript. R.G.L. performed the metabonomics experiments and analyzed the data. Y.Y.X. and Z.L.W. performed the GC-TOF-MS and metabolomics 
analyses. Y.Y.Z. assisted with the in vivo infection trial and ROS measurements. S.D.F assisted in experiments related to extracellular flux analysis. X.G.L aided in the detection of relative enzyme activities and in the ELISAs. X.G.H. provided advice for the in vivo studies. Z.H.L. and Y. L. Y provided guidance and advice. Y.Y.X. provided advice and reagents and oversaw a portion of the work. J.F.M. conceived ideas and oversaw the research program.

\section{DECLARATION OF INTERESTS}

The authors declare no competing interests.

\section{References}

1. Rowe, S. M., S. M. Godden, D. V. Nydam, P. J. Gorden, A. Lago, A. K. Vasquez, E. Royster, J. Timmerman, and M. J. Thomas. 2020. Randomized controlled non-inferiority trial investigating the effect of 2 selective dry-cow therapy protocols on antibiotic use at dry-off and dry period intramammary infection dynamics. J Dairy Sci 103:6473-6492. https://doi.org/10.3168/jds.201917728.

2. Cheng, J., W. Qu, H. W. Barkema, D. B. Nobrega, J. Gao, G. Liu, J. De Buck, J. P. Kastelic, H. Sun, and B. Han. 2019. Antimicrobial resistance profiles of 5 common bovine mastitis pathogens in large Chinese dairy herds. J Dairy Sci 102:2416-2426. https://doi.org/10.3168/jds.2018-15135.

3. Hoque, M. N., A. Istiaq, R. A. Clement, K. M. Gibson, O. Saha, O. K. Islam, R. A. Abir, M. Sultana, A. Z. Siddiki, K. A. Crandall, and M. A. Hossain. 2020. Insights into the resistome of bovine clinical mastitis microbiome, a key factor in disease complication. Front Microbiol 11:860. https://doi.org/10.3389/fmicb.2020.00860.

4. Antok, F. I., R. Mayrhofer, H. Marbach, J. C. Masengesho, H. Keinprecht, V. Nyirimbuga, O. Fischer, S. Lepuschitz, W. Ruppitsch, M. Ehling-Schulz, A. T. Fessler, S. Schwarz, S. Monecke, R. Ehricht, T. Grunert, J. Spergser, and I. Loncaric. 2019. Characterization of antibiotic and biocide resistance genes and virulence factors of Staphylococcus species associated with bovine mastitis in Rwanda. Antibiotics (Basel) 9. https://doi.org/10.3390/antibiotics9010001.

5. Hillerton, J. E., and E. A. Berry. 2005. Treating mastitis in the cow-a tradition or an archaism. J Appl Microbiol 98:1250-1255. https://doi.org/10.1111/j.1365-2672.2005.02649.x.

6. Kessel, S., and C. E. Wittenberg. 2008. [Joint infection in a young patient caused by Streptococcus uberis, a pathogen of bovine mastitis--a case report]. Z Orthop Unfall 146:507-509. https://doi.org/10.1055/s-2007-989352.

7. Almeida, R. A., J. R. Dunlap, and S. P. Oliver. 2010. Binding of host factors influences internalization and intracellular trafficking of Streptococcus uberis in bovine mammary epithelial cells. Vet Med Int 2010:319192. https://doi.org/10.4061/2010/319192.

8. Dai, B., J. Zhang, M. Liu, J. Lu, Y. Zhang, Y. Xu, J. Miao, and Y. Yin. 2016. The role of $\mathrm{Ca}^{2+}$ mediated signaling pathways on the effect of taurine against Streptococcus uberis infection. Vet Microbiol 192:26-33. https://doi.org/10.1016/j.vetmic.2016.06.008. 
9. Zheng, L., Y. Xu, J. Lu, M. Liu, D. Bin, J. Miao, and Y. Yin. 2016. Variant innate immune responses of mammary epithelial cells to challenge by Staphylococcus aureus, Escherichia coli and the regulating effect of taurine on these bioprocesses. Free Radic Biol Med 96:166-180. https://doi.org/10.1016/j.freeradbiomed.2016.04.022.

10. Jin-feng, M., Z. Yuan-shu, H. Guo-qing, M. A. Hai-tian, Z. Si-xiang, and Z. Yu-min. 2009. Polysaccharide Nucleic Acid of Bacillus Calmette Guerin Modulates Th1/Th2 Cytokine Gene Expression in Lipopolysaccharide-Induced Mastitis in Rats. Agricultural Sciences in China 8. https://doi.org/ 10.1016/S1671-2927(08)60308-9.

11. Miao, J., L. Zheng, J. Zhang, Z. Ma, W. Zhu, and S. Zou. 2011. The effect of taurine on the toll-like receptors/nuclear factor kappa B (TLRs/NF-kappaB) signaling pathway in Streptococcus uberisinduced mastitis in rats. Int Immunopharmacol 11:1740-1746.

https://doi.org/10.1016/j.intimp.2011.06.008.

12. Miao, J., J. Zhang, L. Zheng, X. Yu, W. Zhu, and S. Zou. 2012. Taurine attenuates Streptococcus uberis-induced mastitis in rats by increasing T regulatory cells. Amino Acids 42:2417-2428. https://doi.org/10.1007/s00726-011-1047-3.

13. Li, M., P. Xi, Y. Xu, Z. Wang, X. Han, W. Ren, V. Phouthapane, and J. Miao. 2019. Taurine attenuates Streptococcus uberis-induced bovine mammary epithelial cells inflammation via phosphoinositides/Ca ${ }^{2+}$ signaling. Front Immunol 10:1825. https://doi.org/10.3389/fimmu.2019.01825.

14. Li, B., P. Xi, Z. Wang, X. Han, Y. Xu, Y. Zhang, and J. Miao. 2018. PI3K/Akt/mTOR signaling pathway participates in Streptococcus uberis-induced inflammation in mammary epithelial cells in concert with the classical TLRs/NF-kB pathway. Vet Microbiol 227:103-111. https://doi.org/10.1016/j.vetmic.2018.10.031.

15. Z. Wang, R. Lan, Y. Xu, J Zuo, X. Han, V. Phouthapane, Z. Luo, and J. Miao. 2021. Taurine alleviates Streptococcus uberis-induced inflammation by activating autophagy in mammary epithelial cells. Front Immunol. Accepted.

16. Kim, M. J., H. C. Wainwright, M. Locketz, L. G. Bekker, G. B. Walther, C. Dittrich, A. Visser, W. Wang, F. F. Hsu, U. Wiehart, L. Tsenova, G. Kaplan, and D. G. Russell. 2010. Caseation of human tuberculosis granulomas correlates with elevated host lipid metabolism. Embo Mol Med 2:258-274. https://doi.org/10.1002/emmm.201000079.

17. Singh, V., S. Jamwal, R. Jain, P. Verma, R. Gokhale, and K. V. Rao. 2012. Mycobacterium tuberculosisdriven targeted recalibration of macrophage lipid homeostasis promotes the foamy phenotype. Cell Host Microbe 12:669-681. https://doi.org/10.1016/j.chom.2012.09.012.

18. Cumnock, K., A. S. Gupta, M. Lissner, V. Chevee, N. M. Davis, and D. S. Schneider. 2018. Host energy source is important for disease tolerance to malaria. Curr Biol 28:1635-1642. https://doi.org/10.1016/j.cub.2018.04.009.

19. Ribeiro, R. A., M. L. Bonfleur, T. M. Batista, P. C. Borck, and E. M. Carneiro. 2018. Regulation of glucose and lipid metabolism by the pancreatic and extra-pancreatic actions of taurine. Amino Acids 
50:1511-1524. https://doi.org/10.1007/s00726-018-2650-3.

20. Hargrave, K. E., S. Woods, O. Millington, S. Chalmers, G. D. Westrop, and C. W. Roberts. 2019. Multiomics studies demonstrate toxoplasma gondii-induced metabolic reprogramming of murine dendritic cells. Front Cell Infect Microbiol 9:309. https://doi.org/10.3389/fcimb.2019.00309.

21. Smallwood, H. S., S. Duan, M. Morfouace, S. Rezinciuc, B. L. Shulkin, A. Shelat, E. E. Zink, S. Milasta, R. Bajracharya, A. J. Oluwaseum, M. F. Roussel, D. R. Green, L. Pasa-Tolic, and P. G. Thomas. 2017. Targeting metabolic reprogramming by influenza infection for therapeutic intervention. Cell Rep 19:1640-1653. https://doi.org/10.1016/j.celrep.2017.04.039.

22. Tucey, T. M., J. Verma, P. F. Harrison, S. L. Snelgrove, T. L. Lo, A. K. Scherer, A. A. Barugahare, D. R. Powell, R. T. Wheeler, M. J. Hickey, T. H. Beilharz, T. Naderer, and A. Traven. 2018. Glucose homeostasis is important for immune cell viability during candida challenge and host survival of systemic fungal infection. Cell Metab 27:988-1006. https://doi.org/10.1016/j.cmet.2018.03.019.

23. Michalek, R. D., V. A. Gerriets, S. R. Jacobs, A. N. Macintyre, N. J. Maclver, E. F. Mason, S. A. Sullivan, A. G. Nichols, and J. C. Rathmell. 2011. Cutting edge: distinct glycolytic and lipid oxidative metabolic programs are essential for effector and regulatory CD4+ T cell subsets. J Immunol 186:3299-3303. https://doi.org/10.4049/jimmunol.1003613.

24. Rodriguez-Prados, J. C., P. G. Traves, J. Cuenca, D. Rico, J. Aragones, P. Martin-Sanz, M. Cascante, and L. Bosca. 2010. Substrate fate in activated macrophages: a comparison between innate, classic, and alternative activation. J Immunol 185:605-614. https://doi.org/10.4049/jimmunol.0901698.

25. Swanson, K., S. Gorodetsky, L. Good, S. Davis, D. Musgrave, K. Stelwagen, V. Farr, and A. Molenaar. 2004. Expression of a beta-defensin mRNA, lingual antimicrobial peptide, in bovine mammary epithelial tissue is induced by mastitis. Infect Immun 72:7311-7314.

https://doi.org/10.1128/IAl.72.12.7311-7314.2004.

26. Isobe, N., K. Hosoda, and Y. Yoshimura. 2009. Immunolocalization of lingual antimicrobial peptide (LAP) in the bovine mammary gland. Anim Sci J 80:446-450. https://doi.org/10.1111/j.17400929.2009.00652.x.

27. Garcia, D., and R. J. Shaw. 2017. AMPK: mechanisms of cellular energy sensing and restoration of metabolic balance. Mol Cell 66:789-800. https://doi.org/10.1016/j.molcel.2017.05.032.

28. Saxton, R. A., and D. M. Sabatini. 2017. mTOR signaling in growth, metabolism, and disease. Cell 168:960-976. https://doi.org/10.1016/j.cell.2017.02.004.

29. O'Brien, K. L., and D. K. Finlay. 2019. Immunometabolism and natural killer cell responses. Nat Rev Immunol 19:282-290. https://doi.org/10.1038/s41577-019-0139-2.

30. O'Neill, L., and M. N. Artyomov. 2019. Itaconate: the poster child of metabolic reprogramming in macrophage function. Nat Rev Immunol 19:273-281. https://doi.org/10.1038/s41577-019-0128-5.

31. Russell, D. G., L. Huang, and B. C. VanderVen. 2019. Immunometabolism at the interface between macrophages and pathogens. Nat Rev Immunol 19:291-304. https://doi.org/10.1038/s41577-0190124-9. 
32. Escoll, P., and C. Buchrieser. 2019. Metabolic reprogramming: an innate cellular defence mechanism against intracellular bacteria? Curr Opin Immunol 60:117-123. https://doi.org/10.1016/j.coi.2019.05.009.

33. Kedia-Mehta, N., and D. K. Finlay. 2019. Competition for nutrients and its role in controlling immune responses. Nat Commun 10:2123. https://doi.org/10.1038/s41467-019-10015-4.

34. Kumar, A., K. Pyaram, E. L. Yarosz, H. Hong, C. A. Lyssiotis, S. Giri, and C. H. Chang. 2019. Enhanced oxidative phosphorylation in NKT cells is essential for their survival and function. Proc Natl Acad Sci U S A 116:7439-7448. https://doi.org/10.1073/pnas.1901376116.

35. O'Neill, L. A., and D. G. Hardie. 2013. Metabolism of inflammation limited by AMPK and pseudostarvation. Nature 493:346-355. https://doi.org/10.1038/nature11862.

36. Weichhart, T., M. Hengstschlager, and M. Linke. 2015. Regulation of innate immune cell function by mTOR. Nat Rev Immunol 15:599-614. https://doi.org/10.1038/nri3901.

37. Pucino, V., M. Certo, V. Bulusu, D. Cucchi, K. Goldmann, E. Pontarini, R. Haas, J. Smith, S. E. Headland, K. Blighe, M. Ruscica, F. Humby, M. J. Lewis, J. J. Kamphorst, M. Bombardieri, C. Pitzalis, and C. Mauro. 2019. Lactate buildup at the site of chronic inflammation promotes disease by inducing CD4+ T cell metabolic rewiring. Cell Metab 30:1055-1074. https://doi.org/10.1016/j.cmet.2019.10.004.

38. Andrejeva, G., and J. C. Rathmell. 2017. Similarities and distinctions of cancer and immune metabolism in inflammation and tumors. Cell Metab 26:49-70. https://doi.org/10.1016/j.cmet.2017.06.004.

39. Lachmandas, E., L. Boutens, J. M. Ratter, A. Hijmans, G. J. Hooiveld, L. A. Joosten, R. J. Rodenburg, J. A. Fransen, R. H. Houtkooper, R. van Crevel, M. G. Netea, and R. Stienstra. 2016. Microbial stimulation of different Toll-like receptor signalling pathways induces diverse metabolic programmes in human monocytes. Nat Microbiol 2:16246. https://doi.org/10.1038/nmicrobiol.2016.246.

40. O'Neill, L. A., R. J. Kishton, and J. Rathmell. 2016. A guide to immunometabolism for immunologists. Nat Rev Immunol 16:553-565. https://doi.org/10.1038/nri.2016.70.

41. Berger, C. N., V. F. Crepin, T. I. Roumeliotis, J. C. Wright, D. Carson, M. Pevsner-Fischer, R. Furniss, G. Dougan, M. Dori-Bachash, L. Yu, A. Clements, J. W. Collins, E. Elinav, G. J. Larrouy-Maumus, J. S. Choudhary, and G. Frankel. 2017. Citrobacter rodentium subverts ATP flux and cholesterol homeostasis in intestinal epithelial cells in vivo. Cell Metab 26:738-752. https://doi.org/10.1016/j.cmet.2017.09.003.

42. Yang, Y., X. Q. Wu, W. X. Li, H. M. Huang, H. D. Li, X. Y. Pan, X. F. Li, C. Huang, X. M. Meng, L. Zhang, X. W. Lv, H. Wang, and J. Li. 2018. PSTPIP2 connects DNA methylation to macrophage polarization in CCL4-induced mouse model of hepatic fibrosis. Oncogene 37:6119-6135. https://doi.org/10.1038/s41388-018-0383-0.

43. Wang, H., J. Zhu, Z. Liu, H. Lv, P. Lv, F. Chen, J. Fu, Y. Hou, R. Zhao, Y. Xu, Q. Zhang, and J. Pi. 2018. Silencing of long isoforms of nuclear factor erythroid 2 like 1 primes macrophages towards M1 polarization. Free Radic Biol Med 117:37-44. https://doi.org/10.1016/j.freeradbiomed.2018.01.022. 
44. Ho, W. E., Y. J. Xu, C. Cheng, H. Y. Peh, S. R. Tannenbaum, W. Wong, and C. N. Ong. 2014. Metabolomics reveals inflammatory-linked pulmonary metabolic alterations in a murine model of house dust mite-induced allergic asthma. J Proteome Res 13:3771-3782. https://doi.org/10.1021/pr5003615.

45. Vats, D., L. Mukundan, J. I. Odegaard, L. Zhang, K. L. Smith, C. R. Morel, R. A. Wagner, D. R. Greaves, P. J. Murray, and A. Chawla. 2006. Oxidative metabolism and PGC-1 beta attenuate macrophagemediated inflammation. Cell Metab 4:13-24. https://doi.org/10.1016/j.cmet.2006.05.011.

46. Huang, S. C., B. Everts, Y. Ivanova, D. O'Sullivan, M. Nascimento, A. M. Smith, W. Beatty, L. LoveGregory, W. Y. Lam, C. M. O'Neill, C. Yan, Du H, N. A. Abumrad, J. J. Urban, M. N. Artyomov, E. L. Pearce, and E. J. Pearce. 2014. Cell-intrinsic lysosomal lipolysis is essential for alternative activation of macrophages. Nat Immunol 15:846-855. https://doi.org/10.1038/ni.2956.

47. Lambert, I. H., D. M. Kristensen, J. B. Holm, and O. H. Mortensen. 2015. Physiological role of taurine-from organism to organelle. Acta Physiol (Oxf) 213:191-212. https://doi.org/10.1111/apha.12365.

48. Nakaya, Y., A. Minami, N. Harada, S. Sakamoto, Y. Niwa, and M. Ohnaka. 2000. Taurine improves insulin sensitivity in the Otsuka Long-Evans Tokushima Fatty rat, a model of spontaneous type 2 diabetes. Am J Clin Nutr 71:54-58. https://doi.org/10.1093/ajcn/71.1.54.

49. Weinstein, S. L., A. J. Finn, S. H. Dave, F. Meng, C. A. Lowell, J. S. Sanghera, and A. L. DeFranco. 2000. Phosphatidylinositol 3-kinase and mTOR mediate lipopolysaccharide-stimulated nitric oxide production in macrophages via interferon-beta. J Leukoc Biol 67:405-414. https://doi.org/10.1002/jlb.67.3.405.

50. Gou, H., M. Zhao, J. Yuan, H. Xu, H. Ding, and J. Chen. 2017. Metabolic profiles in cell lines infected with classical swine fever virus. Front Microbiol 8:691. https://doi.org/10.3389/fmicb.2017.00691.

\section{Figures}



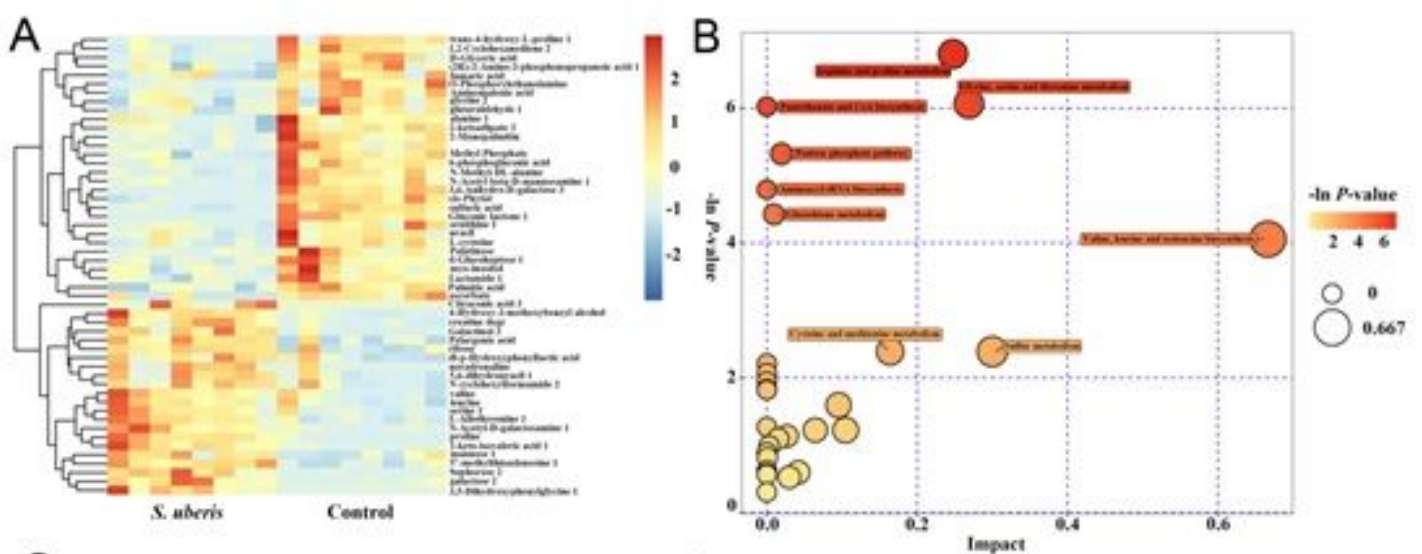

C

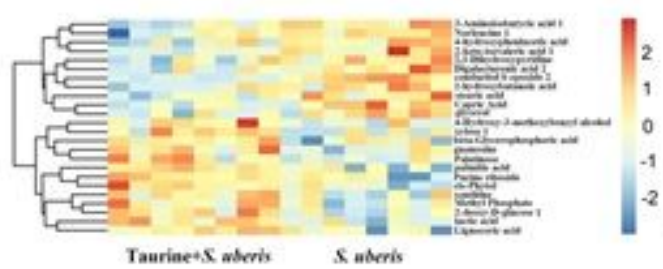

$\mathrm{D}$
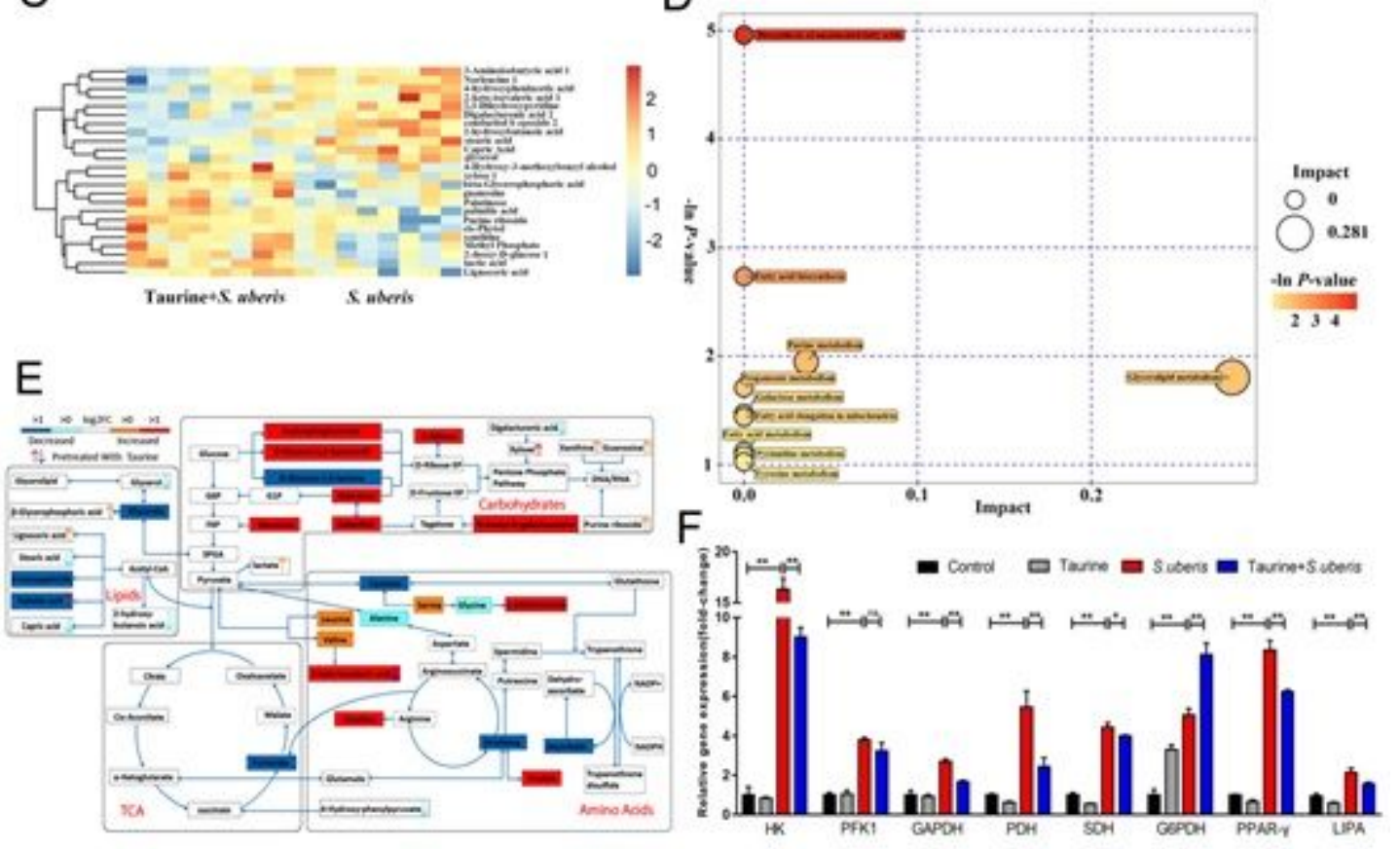

Figure 1

Taurine relieves S. uberis-induced metabolic changes in mammary glands. Pregnant C57BL/6J mice were administered $100 \mathrm{mg} / \mathrm{kg}$ taurine (in sterile pyrogen-free saline) or an equal volume of saline by daily gavage until parturition. At $72 \mathrm{~h}$ post-parturition, mice in the $\mathrm{S}$. uberis and Taurine $+\mathrm{S}$. uberis groups were infused with 100 colony-forming units (CFUs) of S. uberis in $50 \mu \mathrm{L}$ sterile saline into the left 4 th (L4) and right 4th (R4) teats. At $24 \mathrm{~h}$ post-S. uberis-infusion (PI), mammary glands were collected for gas chromatography-time-of-flight-mass spectrometry (GC-TOF-MS) analysis. Eight mice of each group were sampled $(n=8)$. (A) Significant changes in metabolites in C57BL/6J mice mammary glands (S. uberis group versus control group) are shown in the heatmap. (B) Metabolome map of significant metabolic pathways of mammary gland metabolites (S. uberis group versus control group). The $\mathrm{x}$-axis represents pathway enrichment, and the y-axis represents the pathway impact. Large sizes and dark colors represent major pathway-enrichment and high pathway-impact values, respectively. (C) Significant metabolic changes in C57BL/6J mice mammary glands are represented in the heatmap (Taurine $+\mathrm{S}$. uberis group versus S. uberis group). (D) Metabolome map of significant metabolic pathways as indicated by 
metabolites in C57BL/6J mice mammary tissues (Taurine $+\mathrm{S}$. uberis group versus $\mathrm{S}$. uberis group). (E) Model of how taurine changes metabolomic responses to $\mathrm{S}$. uberis infection in C57BL/6J mice mammary glands. (F) Relative gene expression of mammary gland samples from different groups. Data are represented as mean \pm SEM $(n=3)$. ${ }^{*} P<0.05$.
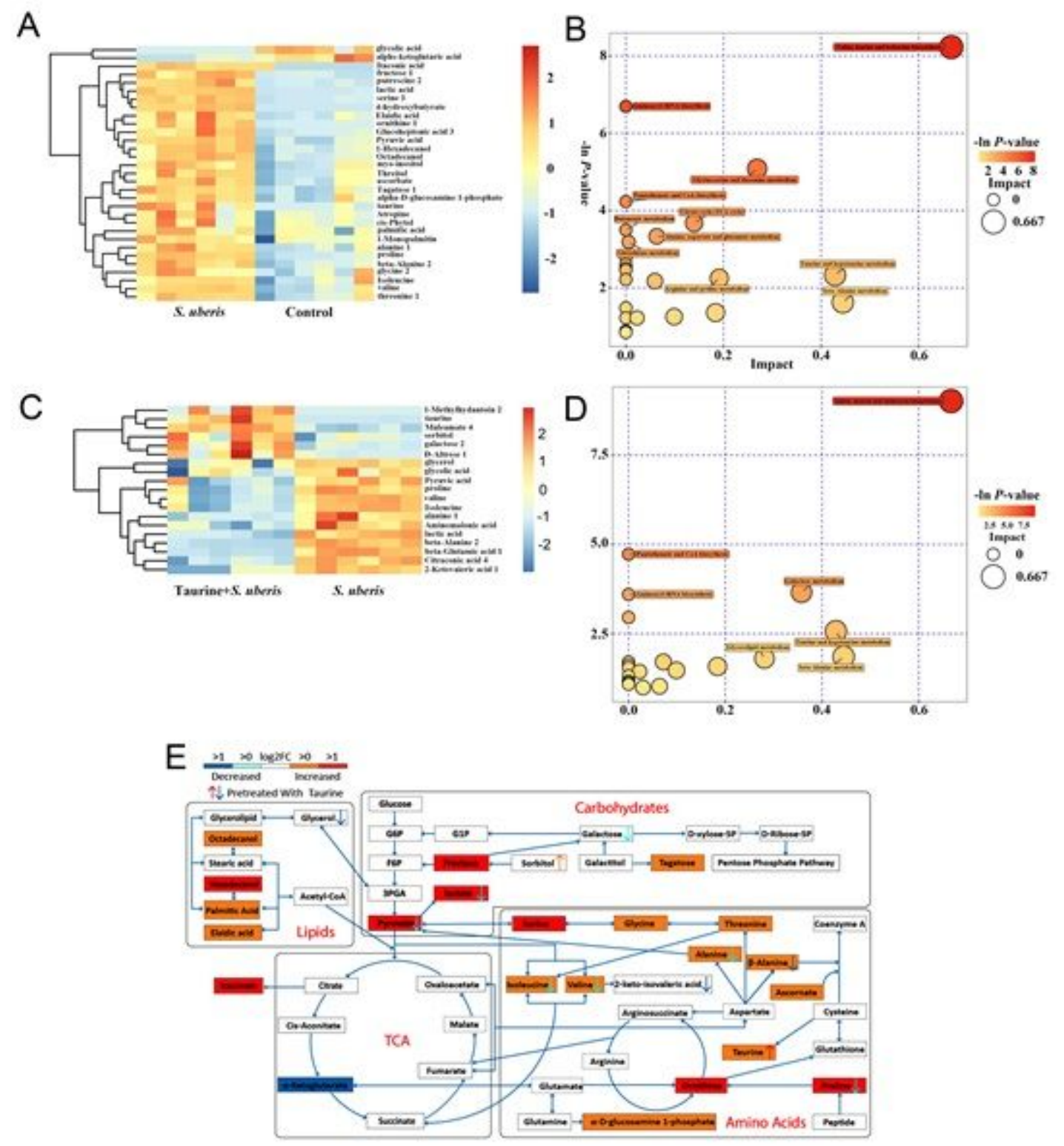

\section{Figure 2}

Taurine attenuates metabolic disturbances in S. uberis infected MECs. EpH4-Ev cells were pretreated with taurine for $24 \mathrm{~h}$ and infected with S. uberis in mid-exponential phase at a multiplicity of infection (MOI) of 10 for $3 \mathrm{~h}$ at $37^{\circ} \mathrm{C}$. Cellular metabolites were extracted and assayed by GC-TOF-MS. Six samples from each group were tested $(n=6)$. (A) Significant changes in metabolites in EpH4-Ev cells (S. uberis group versus control group) are shown in the heatmap. (B) Metabolome map of significant metabolic pathways reflected by metabolites of EpH4-Ev cells (S. uberis group versus control group). The x-axis represents pathway enrichment, and the $y$-axis represents the pathway impact. Large sizes and dark colors represent major-pathway enrichment and high pathway-impact values, respectively. (C) Significant metabolic 
changes in EpH4-Ev cells (Taurine $+\mathrm{S}$. uberis group versus S. uberis group) are shown. (D) Metabolome map of significant metabolic pathways identified by metabolites in EpH4-Ev cells (Taurine $+\mathrm{S}$. uberis group versus S. uberis group). (E) Model of how taurine alters the metabolomic response of in vitro EpH4Ev cells in response to a $3 \mathrm{~h} \mathrm{~S}$. uberis infection.
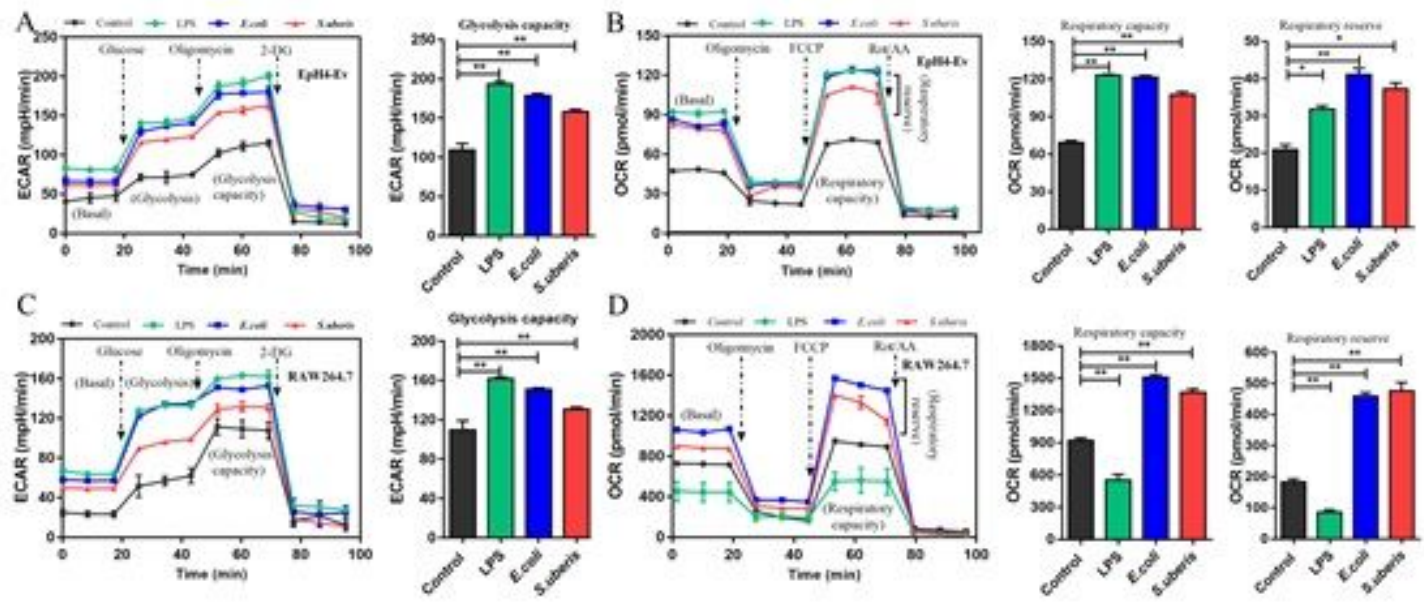

\section{Figure 3}

Metabolic responses in MECs and macrophages to various microbial stimuli. (A-B) Stimulation with 10 $\mu \mathrm{g} / \mathrm{mL}$ LPS for $12 \mathrm{~h}$ or inactivated S. uberis or E. coli $(\mathrm{MOI}=100)$ for $3 \mathrm{~h}$ at $37^{\circ} \mathrm{C}$. Real-time changes in ECAR (A) and OCR (B) in EpH4-Ev cells were determined. (C-D) Stimulation with $100 \mathrm{ng} / \mathrm{mL}$ LPS for $12 \mathrm{~h}$ or inactivated $\mathrm{S}$. uberis or E. coli $(\mathrm{MOI}=5)$ for $3 \mathrm{~h}$ at $37^{\circ} \mathrm{C}$. Real-time changes in ECAR (C) and OCR (D) in RAW264.7 cells were determined. Data are represented as mean \pm SEM $(n=3)$. ${ }^{*} P<0.05$. 

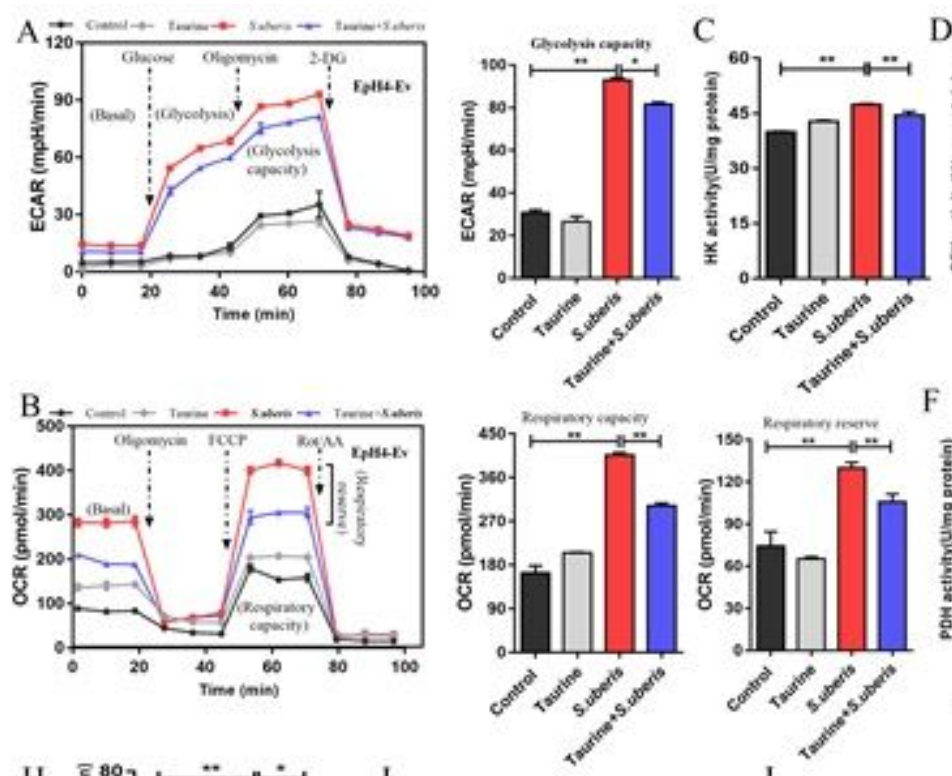

$\mathrm{F} \quad \mathrm{G}$
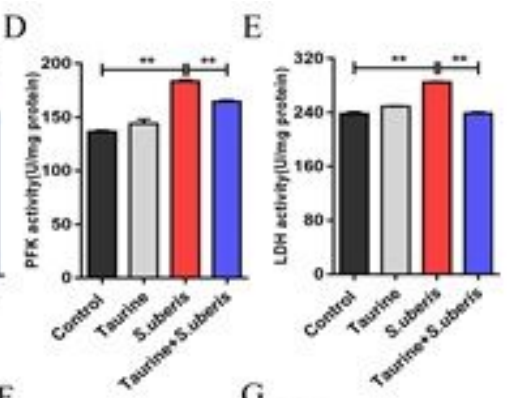

$\mathrm{H}$

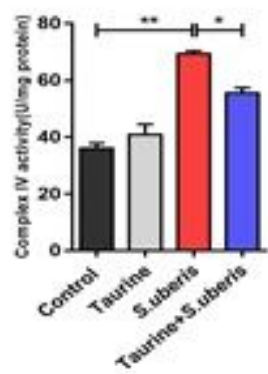

I

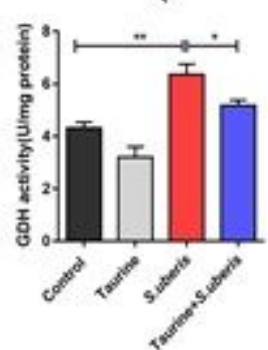

$\mathrm{J}$

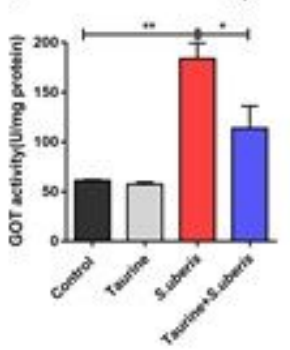

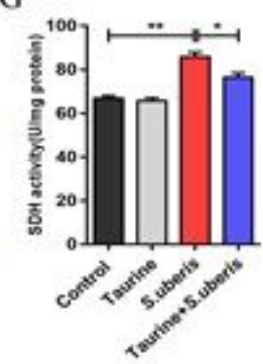

K

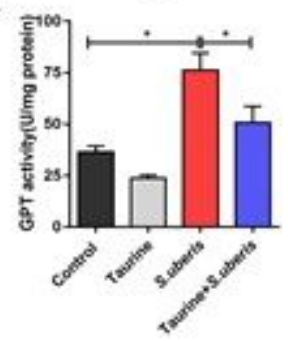

\section{Figure 4}

Taurine reprograms cellular metabolism by coordinaing the energy supply and anabolic intermediate production. (A-B) EpH4-Ev cells were pretreated with taurine for $24 \mathrm{~h}$ and then stimulated with inactivated S. uberis $(\mathrm{MOI}=100)$ for $3 \mathrm{~h}$ at $37^{\circ} \mathrm{C}$. Real-time changes in ECAR $(A)$ and OCR $(B)$ in EpH4-Ev cells were assessed during sequential treatment with oligomycin, carbonyl cyanide-4-(trifluoromethoxy) phenylhydrazone (FCCP), and antimycin A + rotenone. (C-H) After taurine pretreatment, EpH4-Ev cells were infected with S. uberis in mid-exponential phase $(\mathrm{MOI}=10)$ for $3 \mathrm{~h}$ at $37^{\circ} \mathrm{C}$. Enzyme activities related to glycolysis and OXPHOS were determined using commercial kits. (I-K) EpH4-Ev cells were pretreated with taurine for $24 \mathrm{~h}$ and then infected in mid-exponential phase for $3 \mathrm{~h}$ at $37^{\circ} \mathrm{C}$ with $\mathrm{S}$. uberis $(\mathrm{MOI}=10)$. The activities of enzymes linking amino acid and energy-related metabolites were determined using commercial kits. Data are represented as mean $\pm \operatorname{SEM}(n=3) .{ }^{*} P<0.05$. 

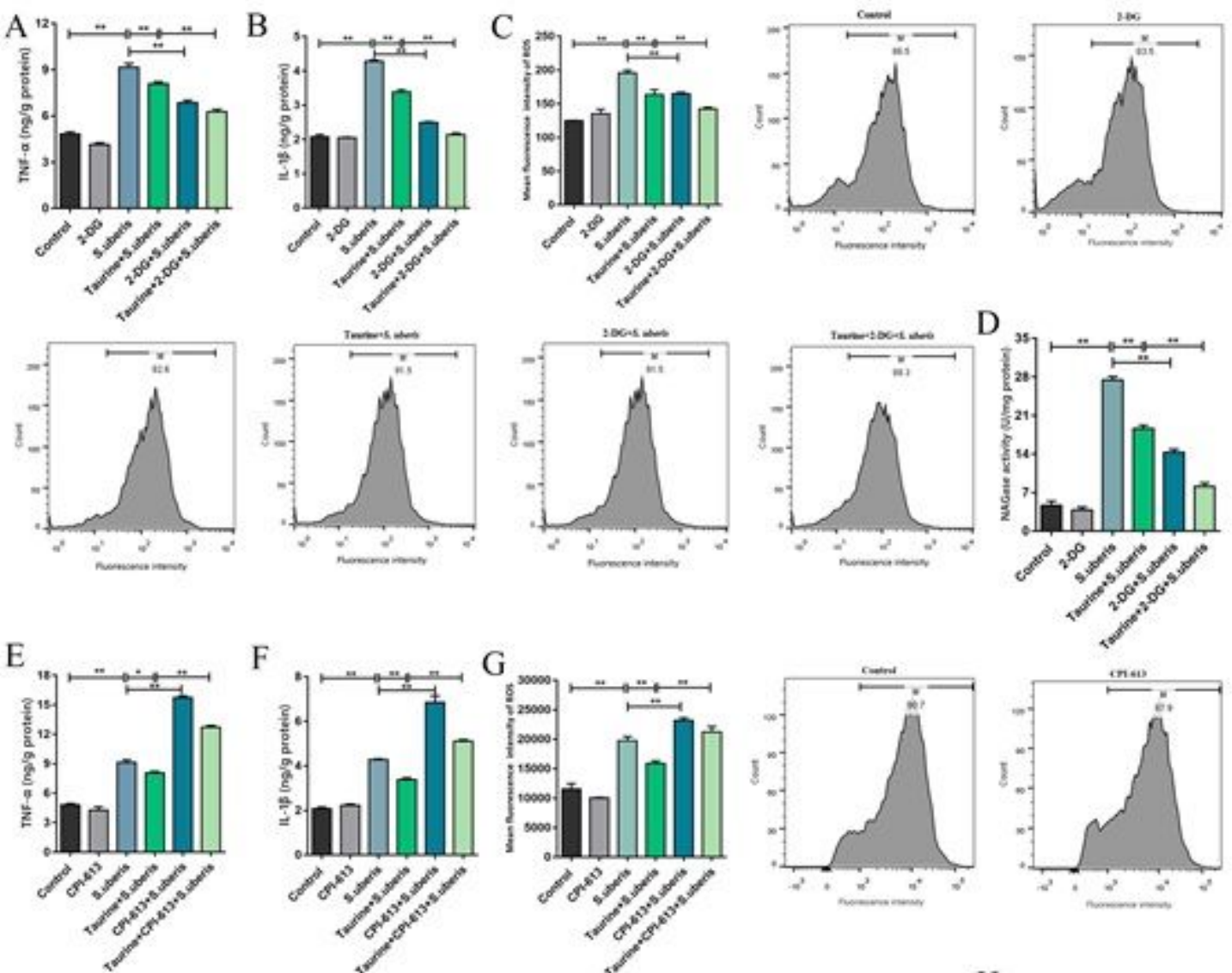

$\mathrm{F}$
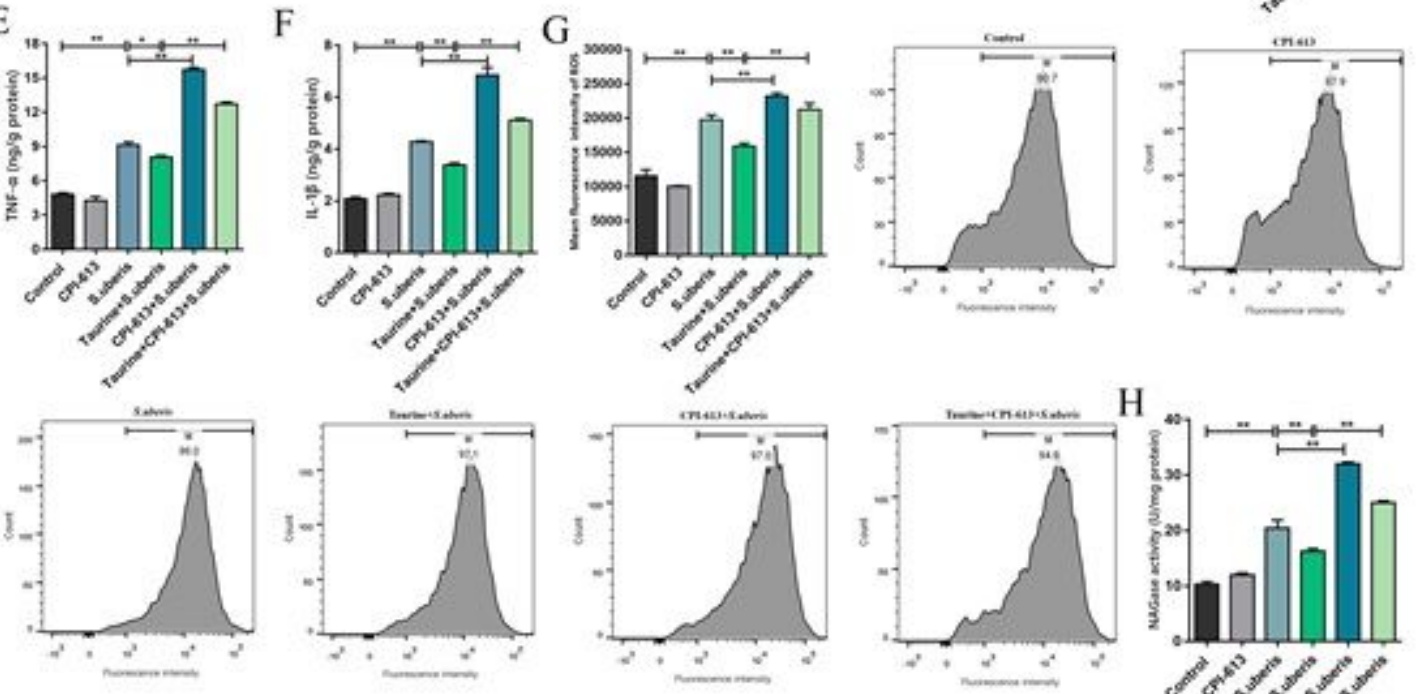
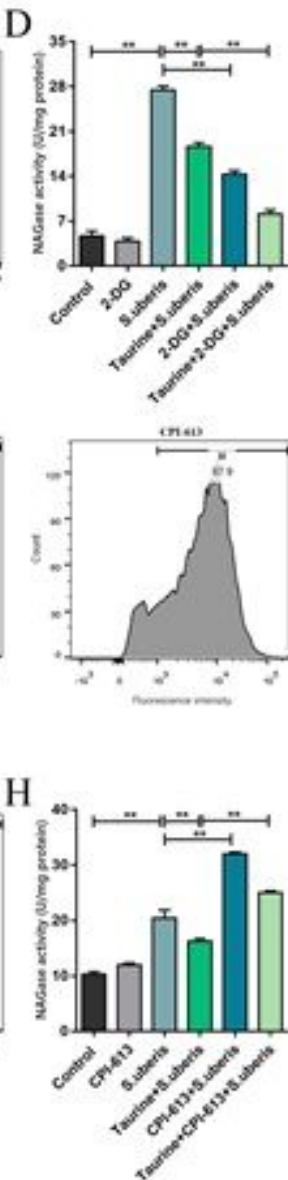

\section{Figure 5}

Taurine balance metabolism to alleviate inflammation induced by S. uberis. EpH4-Ev cells were pretreated with taurine for $24 \mathrm{~h}$ and infected with $\mathrm{S}$. uberis in mid-exponential phase $(\mathrm{MOI}=10)$ for $3 \mathrm{~h}$ at $37^{\circ} \mathrm{C}$. The cells were treated with $5 \mathrm{mM}$ 2-DG for $1 \mathrm{~h}$ to block glycolysis or with $25 \mu \mathrm{M} \mathrm{CPI}-613$ for $12 \mathrm{~h}$ to block the TCA cycle prior to $S$. uberis infection. (A, B, K, L) TNF- $\alpha$ and IL-1 $\beta$ levels in the supernatants of EpH4-Ev cells pretreated with 2-DG $(\mathrm{A}, \mathrm{B})$ or $\mathrm{CPI}-613(\mathrm{~K}, \mathrm{~L})$ were measured using enzyme-linked immunosorbent assay (ELISA) kits. (C-I, M-S) Intracellular ROS content was evaluated by staining cells $(10,000$ cells/sample) with DCFH-DA, followed by analysis using CellQuest Pro acquisition and FlowJo software. $(J, T)$ NAGase activity in the supernatants. Data are represented as mean $\pm S E M(n=3)$. ${ }^{*}<0.05$. 
A
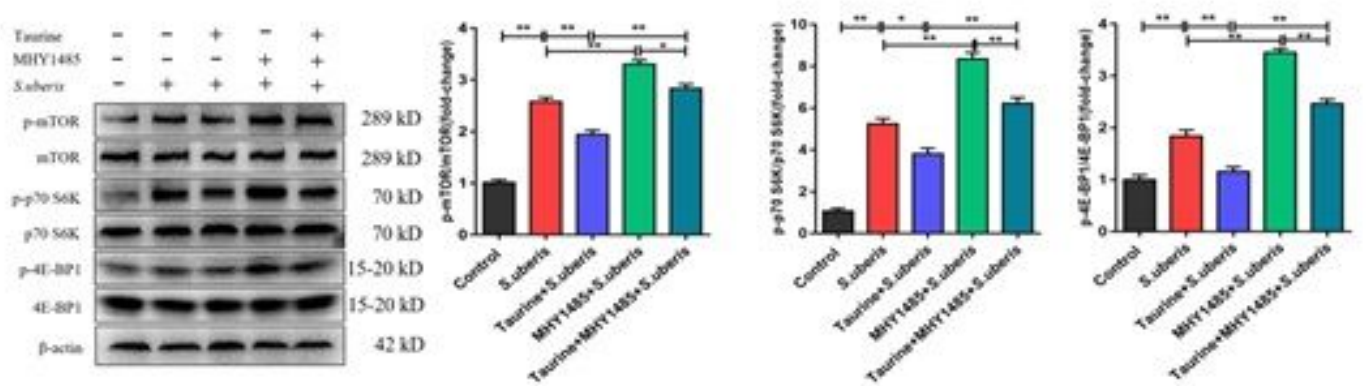

B
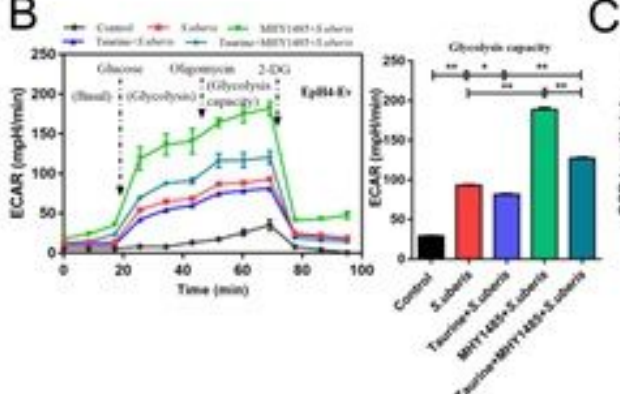

$\mathrm{C}$
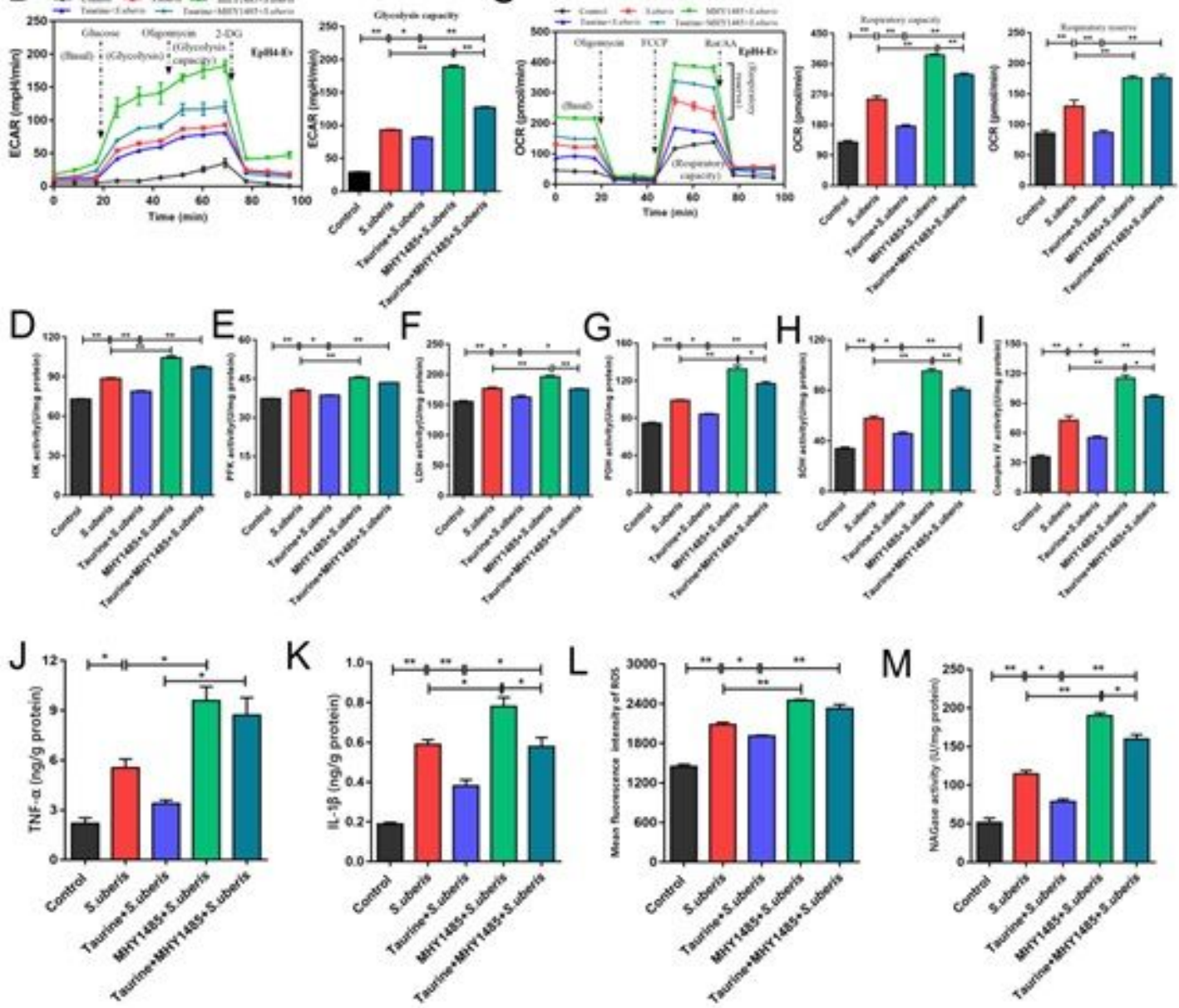

Figure 6

Taurine regulates metabolic alterations in S. uberis infection by inhibiting the mTOR pathway. (A) EpH4Ev cells were pretreated with taurine for $24 \mathrm{~h}$ and infected with $\mathrm{S}$. uberis in mid-exponential phase ( $\mathrm{MOI}=$

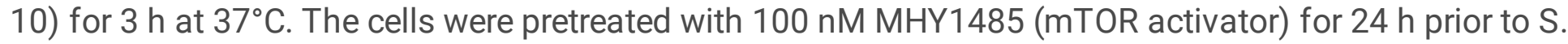
uberis infection. The protein-expression levels of mTOR, p70 S6K, and 4E-BP1, as well as the levels of each phosphorylated protein ( $\mathrm{p}-\mathrm{mTOR}, \mathrm{p}-\mathrm{p} 70 \mathrm{~S} 6 \mathrm{~K}$, and p-4EBP1) were determined by western blotting. (BC) EpH4-Ev cells were pretreated with taurine for $24 \mathrm{~h}$ and then stimulated with inactivated S. uberis (MOI $=100$ ) for $3 \mathrm{~h}$ at $37^{\circ} \mathrm{C}$. The cells were pretreated with $100 \mathrm{nM} \mathrm{MHY} 1485$ for $24 \mathrm{~h}$ before $\mathrm{S}$. uberis infection. Real-time changes in the ECAR (B) and OCR (C) levels in EpH4-Ev cells were determined. (D-I) The relative activities of enzymes driving glycolysis and OXPHOS were determined using commercial kits. (J-K) TNF- $\alpha$ and IL-1 $\beta$ levels in EpH4-Ev cell supernatants pretreated with $100 \mathrm{nM} \mathrm{MHY1485}$ were 
measured by ELISA. (L) Intracellular ROS contents were evaluated by staining cells (10,000/sample) with DCFH-DA, followed by analysis using CellQuest Pro acquisition and FlowJo software. (M) Supernatant NAGase activities were determined using commercial kits. Data are represented as mean \pm SEM $(n=3)$. ${ }^{*}$ $<0.05$.

A
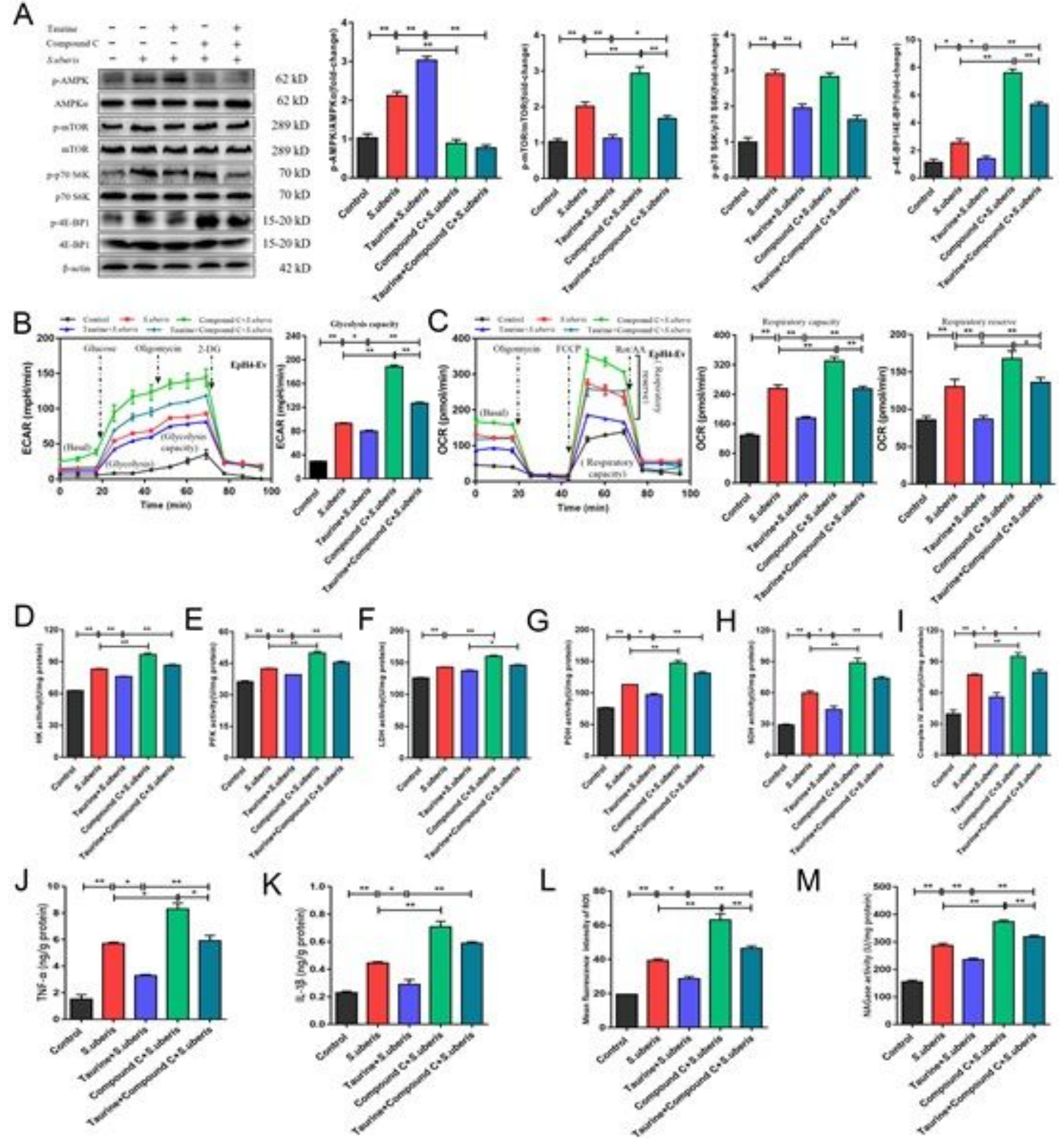

$\mathrm{L}$

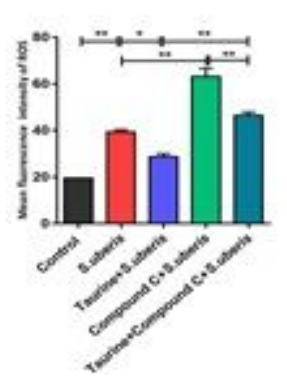

$\mathrm{M}$

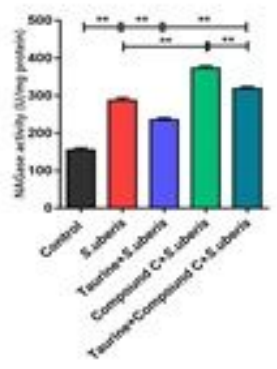

Figure 7

Taurine reverses mTOR-mediated metabolic alterations in MECs via AMPK. (A) EpH4-Ev cells were pretreated with taurine for $24 \mathrm{~h}$ and infected with $\mathrm{S}$. uberis in mid-exponential phase $(\mathrm{MOI}=10)$ for $3 \mathrm{~h}$ at $37^{\circ} \mathrm{C}$. Cells were then further pretreated with $10 \mu \mathrm{M}$ compound $\mathrm{C}$ (an AMPK inhibitor) for $1 \mathrm{~h}$ before S. uberis infection. The protein-expression levels of AMPKa, mTOR, p70 S6K, and 4E-BP1, and the corresponding phosphorylated proteins (p-AMPK, p-mTOR, p-p70 S6K, and p-4EBP1) were assayed by western blot. (B-C) EpH4-Ev cells were pretreated with taurine for $24 \mathrm{~h}$ and then with $10 \mu \mathrm{M}$ Compound $\mathrm{C}$ prior to stimulation with inactivated $\mathrm{S}$. uberis $(\mathrm{MOI}=100)$ for $3 \mathrm{~h}$ at $37^{\circ} \mathrm{C}$. Real-time changes in ECAR $(B)$ and OCR (C) levels in EpH4-Ev cells were determined. (D-I) The relative activities of enzymes related to 
glycolysis and OXPHOS were determined using commercial kits. (J-K) TNF- $a$ and IL-1 $\beta$ production in the supernatants of EpH4-Ev cells pretreated with $10 \mu \mathrm{M}$ Compound $\mathrm{C}$ were measured via ELISA. (L) Intracellular ROS contents were evaluated by staining cells with DCFH-DA, followed by analysis $(10,000$ cells/sample) with CellQuest Pro acquisition and FlowJo software. (M) Supernatant NAGase activities were determined using commercial kits. Data are represented as mean $\pm \operatorname{SEM}(n=3)$. ${ }^{*}<0.05$.

\section{Supplementary Files}

This is a list of supplementary files associated with this preprint. Click to download.

- SupplementaryFigures.docx 